\title{
Post-glacial hunter-gatherer subsistence patterns in Britain: dietary reconstruction using FRUITS
}

\author{
Catriona Pickard $^{1}$ (D) Clive Bonsall $^{1}$
}

Received: 17 January 2020 / Accepted: 20 May 2020/Published online: 25 June 2020

(C) The Author(s) 2020

\begin{abstract}
The diets of 85 individuals from 21 sites were modelled using FRUITS based on their bulk bone collagen $\mathrm{C}$ and $\mathrm{N}$ isotope ratio signatures. The sites, which occur in a range of environments, group into three distinct periods corresponding to the British 'Late Upper Palaeolithic', 'Early Mesolithic' and 'Late Mesolithic', respectively. The FRUITS models for three LUP sites dated to the Bølling-Allerød Interstadial suggest an emphasis on terrestrial (animal and plant) resources. The FRUITS predictions for the Early and Late Mesolithic suggest there was significant variability in diet between sites and occasionally between individuals from the same site. The Late Mesolithic coastal site of Cnoc Coig in western Scotland shows the expected emphasis on marine resources. In contrast, Early and Late Mesolithic coastal sites in South Wales show greater reliance on terrestrial food sources. In several cases, our model predictions differ from the interpretations of previous authors. A surprising outcome is the lack of evidence for the consumption of freshwater resources at sites near large rivers. We add the caveat that our model predictions are likely influenced by inadequate baseline $\delta^{13} \mathrm{C}$ and $\delta^{15} \mathrm{~N}$ data for wild terrestrial plant and aquatic resources, in particular.
\end{abstract}

Keywords FRUITS $\cdot$ Stable isotopes $\cdot$ Late Upper Palaeolithic $\cdot$ Mesolithic $\cdot$ Britain

\section{Introduction}

After more than a century of archaeological investigation, knowledge of the lifeways of post-glacial hunter-gatherers in Britain is still extremely limited. In large part, this reflects the generally poor preservation of food remains in open-air archaeological sites (especially animal bones) due to adverse soil conditions, as well as the inundation of coastal areas by relative sea-level rise since the Last Glacial Maximum - the effects of which have been exacerbated by inadequate archaeological recovery techniques and regional research biases.

This article is part of the Topical Collection on Post-glacial human subsistence and settlement patterns

Electronic supplementary material The online version of this article (https://doi.org/10.1007/s12520-020-01087-1) contains supplementary material, which is available to authorized users.

Catriona Pickard

Catriona.Pickard@ed.ac.uk

1 School of History, Classics and Archaeology, University of Edinburgh, Old Medical Quad, Teviot Place, Edinburgh EH8 9AG, UK
In consequence, knowledge of post-glacial hunter-gatherer subsistence patterns relies heavily on stable isotope analysis of human remains found mainly in the relatively protected environment of caves. Here, we present a synthesis of the bone collagen stable isotope data for British hunter-gatherer populations, evaluate the robustness of conventional approaches to interpreting stable isotope data and offer new interpretations of hunter-gatherer diet using Bayesian mixing models.

\section{Palaeodiet and stable isotope analysis}

Stable isotope analysis of bone collagen is a long-established tool for reconstructing past diet (see Schoeninger 2010 for a review). The principles of stable isotope interpretation were established in the 1970s and 1980s (Vogel and van der Merwe 1977; Schoeninger and DeNiro 1984). Carbon $\left(\delta^{13} \mathrm{C}\right)$ and nitrogen $\left(\delta^{15} \mathrm{~N}\right)$ isotope ratios in the tissues of humans and other mammals reflect those of foods consumed. Carbon is incorporated into plant tissues during photosynthesis. Carbon stable isotope ratios of plants vary depending on the environmental sources of carbon and the fixation mechanism used. Most plants that constitute a significant part of human foodwebs fix carbon through one of two photosynthetic 
routes, either the $\mathrm{C}_{3}$ or the $\mathrm{C}_{4}$ pathway (DeNiro and Epstein 1978). $C_{4}$ plants were a very minor component of the Northwest European post-glacial flora (cf. Long 1983) and, although some species are edible, they are unlikely to have contributed significantly to the diets of Lateglacial and Holocene hunter-gatherers. Most marine plants and temperate region grasses (including wild and domestic varieties of cereals such as wheat, barley, oats and rye), as well as most fruits and vegetables, have $\mathrm{C}_{3}$ cycles. Terrestrial $\mathrm{C}_{3}$ plants, which fix carbon from atmospheric $\mathrm{CO}_{2}$, have lower average $\delta^{13} \mathrm{C}$ (c. $-26.5 \%$ ) than marine plants owing to distinct environmental carbon sources - marine plants fix carbon from oceanic carbonate as well as $\mathrm{CO}_{2}$, which is relatively ${ }^{13} \mathrm{C}$-enriched $\left(+7 \%\right.$ o in comparison to atmospheric $\mathrm{CO}_{2}$ ) (e.g. Maberly et al. 1992). Variations in plant $\delta^{13} \mathrm{C}$ are passed on through respective food chains into the tissues of human consumers. There is an offset or fractionation of c. $+5 \%$ o between plants and consumers, i.e. human consumers of terrestrial $\mathrm{C}_{3}$ resources typically have $\delta^{13} \mathrm{C}$ values of c. $-21.5 \%$, while human consumers of marine resources typically have $\delta^{13} \mathrm{C}$ values of c. $-13.0 \%$ (Chisholm et al. 1982; Tykot 2004). Measurement of bone collagen $\delta^{13} \mathrm{C}$ can thus indicate the relative proportions of terrestrial versus marine food sources in diet.

Notably, the $\delta^{13} \mathrm{C}$ values of freshwater food sources may overlap with those of terrestrial $\mathrm{C}_{3}$ food webs (e.g. Bonsall et al. 1997), particularly in temperate regions (i.e. with $C_{3}$ vegetation in the watershed) and water systems where the main source of dissolved inorganic carbon is atmospheric $\mathrm{CO}_{2}$ (Finlay and Kendall 2007).

Co-analysis of both $\delta^{13} \mathrm{C}$ and $\delta^{15} \mathrm{~N}$ allows additional dietary discrimination. Nitrogen in plants may be assimilated with little fractionation from atmospheric $\mathrm{N}_{2}$ as well as from soil (Nadelhoffer and Fry 1994). Plant $\delta^{15} \mathrm{~N}$, therefore, varies according to the environmental source (von Wirén et al. 1997). Metabolic fractionation of nitrogen stable isotopes occurs at each trophic level of the food chain, resulting in 'stepped ${ }^{15} \mathrm{~N}$ enrichment. Within a single biome, plants have lower $\delta^{15} \mathrm{~N}$ values than herbivores, which in turn have lower values than carnivores (DeNiro and Epstein 1981; Katzenberg 2000). The offset between humans and diet has been proposed to be up to c. $6 \%$ ( $\left(\mathrm{O}^{\prime}\right.$ Connell et al. 2012). Measurement of bone collagen $\delta^{15} \mathrm{~N}$ can thus indicate the relative importance of plant versus animal food sources in diet, and by extension the trophic level of the consumer. Additionally, $\delta{ }^{15} \mathrm{~N}$ values enable discrimination between terrestrial and freshwater food sources. Again, the two food sources may be distinguished by $\delta^{15} \mathrm{~N}$ values, which are typically higher in freshwater foods. Elevated $\delta^{15} \mathrm{~N}$ in aquatic foods is a consequence of the larger number of trophic levels in both marine and freshwater food webs and may also result from enrichment through bacterial activity (Schoeninger and DeNiro 1984; Schoeninger 2010).

\section{Dietary models}

Stable isotope data for Lateglacial and Holocene huntergatherers in Britain, while limited, can shed light on aspects of past behaviour, including resource availability, subsistence strategies and mobility. Conventionally, linear models have been employed to quantify the proportions of different foods in diet from carbon and nitrogen stable isotope measurements (e.g. Richards et al. 2000; Bocherens and Drucker 2006; Stevens et al. 2010). However, such models are problematic.

Linear Mixing Models (LMMs) have significant limitations:

- Mathematical constraints limit the number of food sources that can be robustly modelled. Generally, dietary proportions have been quantified from one $\left(\delta^{13} \mathrm{C}\right)$ or two proxies $\left(\delta^{13} \mathrm{C}\right.$ and $\left.\delta^{15} \mathrm{~N}\right)$. However, multiple isotopically distinct food sources are attested in the archaeological record, often greatly exceeding the number of dietary proxies. Where the number of food sources exceeds the number of proxies by more than one, multiple dietary 'solutions' may be generated by LMMs (Phillips et al. 2005; e.g. Bocherens and Drucker 2006) - and these are of limited utility.

- The greater precision offered by combining variables (Phillips et al. 2005) has led to the 'lumping' of food sources and the creation of binary (i.e. terrestrial vs. marine) dietary models of hunter-gatherer diet (e.g. Richards et al. 2005). The result is a dietary model that distinguishes three main categories of diet: (1) marine dependence, (2) terrestrial (i.e. herbivore/omnivore) dependence and (3) mixed terrestrial-marine diets.

- Plant foods are largely overlooked in LMM dietary models. This possibly reflects the perception that plant foods generally are low in protein (e.g. Bownes et al. 2017).

\section{Bayesian modelling of palaeodiet}

In principle, Bayesian mixing models (BMMs) offer more realistic reconstructions of dietary intake than conventional LMMs (Fernandes et al. 2014; Parnell et al. 2014). Uncertainties in trophic level offsets and food-source isotope values, as well as variation in dietary routing and food group elemental composition, can be incorporated into BMMs. The Bayesian mixing model FRUITS-Food Reconstruction Using Isotopic Transferred Signals - is used here to evaluate the relative caloric contribution of multiple food sources to an individual's whole diet (Fernandes et al. 2014, 2015).

Four models of British hunter-gatherer diets were generated using FRUITS: 
MODELS 1 and 2 are protein routed concentrationdependent models, which assume dietary protein was directly routed to bone collagen.

Model 1. Late Upper Palaeolithic (LUP) diets reconstructed using LUP food source stable isotope values.

Model 2. Mesolithic diets reconstructed using Mesolithic food source stable isotope values.

MODELS 3 and 4 are 'nutrient scrambled' (fraction weighted, concentration-dependent) models, which assume collagen carbon was derived from dietary protein and energy sources, in the proportions $74 \pm 4 \%$ and $26 \pm 4 \%$, respectively (cf. Fernandes et al. 2015).

Model 3. LUP diets reconstructed using LUP food source stable isotope values.

Model 4. Mesolithic diets reconstructed using Mesolithic food source stable isotope values.

\section{Palaeodietary model offsets and uncertainties}

Our models use the human diet-to-collagen enrichment factors recommended by Fernandes et al. (2015): $\delta^{13} \mathrm{C}_{\text {diet-collagen }}=+$ $4.8 \pm 0.5 \% \circ$ and $\delta^{15} \mathrm{~N}_{\text {diet-collagen }}=+5.5 \pm 0.5 \%$.

Carbon and nitrogen stable isotope values of food sources are often derived from archaeological bone samples. Generally, however, animal bone is not consumed (although small fish may be consumed whole). There are $\delta^{13} \mathrm{C}$ and $\delta^{15} \mathrm{~N}$ offsets between animal bone and consumed tissues (e.g. muscle and fat). Widely accepted or 'consensus' offsets for terrestrial animals and fish quoted by Fernandes et al. (2015) are used here:

- Terrestrial animals $\Delta^{13} \mathrm{C}_{\text {protein-bone collagen }}=-2.0 \%$ o, and $\Delta^{15} \mathrm{~N}_{\text {protein-bone collagen }}=+2 \%$.

- Terrestrial animals $\Delta^{13} \mathrm{C}_{\text {lipid-bone collagen }}=-8.0 \%$.

- Fish $\Delta^{13} \mathrm{C}_{\text {protein-bone collagen }}=-1.0 \%$, and $\Delta^{15} \mathrm{~N}_{\text {protein-bone }}$ collagen $=+2 \%$.

- Fish $\Delta^{13} \mathrm{C}_{\text {lipid-bone collagen }}=-7.0 \%$.

Shellfish $\Delta{ }^{13} \mathrm{C}_{\text {protein-lipid }}$ is calculated to be $-3.5 \%$ o (from data in Ricca et al. 2007). Studies of the isotope values and offsets in marine mammal tissues are limited (for a review, see Newsome et al. 2010). Offset values between consumed tissue protein and lipids to bone collagen were determined from published offsets between diet and keratin, lipid or muscle values for seal. The $\delta^{13} \mathrm{C}$ offset between keratin and bone collagen is c. $-1.5 \%$ (Bocherens et al. 2014; Crowley et al. 2010). It is assumed there is no significant difference between the $\delta^{15} \mathrm{~N}$ values of keratin and collagen (Bocherens et al. 2014). Variation in the carbon and nitrogen stable isotope ratios of different keratinaceous tissues, e.g. hair, claw and whisker, in seals with uniform diets appear to be small (see Hobson et al. 1996).
- Seal $\Delta^{13} \mathrm{C}_{\text {protein-bone collagen }}=-2.3 \%$, and $\Delta^{15} \mathrm{~N}_{\text {protein-bone }}$ collagen $=-0.6 \%$ o (Hobson et al. 1996).

- Seal $\Delta^{13} \mathrm{C}_{\text {lipid-bone collagen }}=-7.6 \%$ (use of fossil fuels 1996; Germain et al. 2012).

Errors in mass spectrometric measurements introduce uncertainty in consumer $\delta^{13} \mathrm{C}$ and $\delta^{15} \mathrm{~N}$ values. Although measurement error is generally reported to be in the order of \pm $0.1 \%$ of $\delta^{13} \mathrm{C}$ and $\pm 0.2 \%$ for $\delta^{15} \mathrm{~N}$ for the current dietary reconstruction, uncertainty was cautiously set at $0.5 \%$ for both $\delta^{13} \mathrm{C}$ and $\delta^{15} \mathrm{~N}$ following Fernandes et al. $(2014,2015)$. Uncertainty in food source stable isotope values (reflecting differences in preparation methods and seasonal and physiological variations in animal metabolism) were set at $1.0 \%$ o (Fernandes et al. 2014, 2015).

\section{Food group composition}

Establishing the proportional contribution of protein/energy in food sources is essential to the accurate reconstruction of diet (Phillips and Koch 2002). Carbon weight composition (wtC\%) of plant cereals were drawn from Fernandes et al. (2015). Mean composition values for terrestrial mammals, shellfish, fish and sea mammals were calculated from available food composition data from the United States Department of Agriculture (USDA n.d.) Food Composition Databases (https://fdc.nal.usda.gov/), rounded to a multiple of 5 (cf. Fernandes et al. 2015).

Terrestrial animal protein/energy concentration varies significantly depending on the species and also the portion of the animal consumed. For example, the body fat content of deer is highly variable seasonally and individually: fat content of femur marrow in white-tailed deer fawns can exceed $80 \%$, while total body fat ranged from $2.3 \%$ to $48.9 \%$ and protein content from $39.2 \%$ to $75.5 \%$ (Watkins et al. 1991). Wild boar lean muscle, e.g. the tenderloin, has wtC\% protein/energy of c. $85: 15$, while a more fatty portion of the animal, e.g. the belly, has $\mathrm{wtC} \%$ protein/energy of c. 40:60. It is assumed in our models that all edible parts of the animal were consumed. Intermediate wtC\% protein/energy values were used with conservation errors to account for variability in food sources, $\mathrm{wtC} \%$ protein $=60 \pm 5 \%$ and $\mathrm{wtC} \%$ energy $=40 \pm 5 \%$.

The protein content of wild plants is highly variable. Plants with relatively high protein content, such as hazelnuts, may have been important in some post-glacial hunter-gatherer diets. Mushrooms with much lower protein content could also have been important. Plant $\mathrm{wtC} \%$ protein was therefore set at $10 \pm 5 \%$ and $\mathrm{wtC} \%$ energy $=90 \pm 5 \%$.

Defining the lipid/protein carbon weight composition of fish (marine and freshwater) and sea mammals is non-trivial. The lipid content of fish varies significantly both within and between species (e.g. Berg and Bremset 1998; Pinnegar and Polunin 1999); typically $<1 \mathrm{~g} / 100 \mathrm{~g}$ in lean fish such as 
gadids, and $>10 \mathrm{~g} / 100 \mathrm{~g}$ in fatty anadromous species including salmonids and eel (USDA database). Both lean and fatty fish have been identified in the fish assemblages of Lateglacial and Early Holocene sites across Europe (e.g. Pickard and Bonsall 2004; Robson et al. 2016). While freshwater fish are absent from British LUP assemblages and the availability of these resources is debatable (see discussion below), they are relatively common on Lateglacial sites in Continental Europe (e.g. Cleyet-Merle 1990; Bonsall et al. 2016). The mean protein and energy $\mathrm{wtC} \%$ of marine species typically recovered from British hunter-gatherer sites are c. $75 \pm 5 \%$ and $25 \pm 5 \%$ (USDA database), respectively, while those of freshwater fish are c. $65 \pm 5 \%$ and $35 \pm 5 \%$ (USDA database; Fernandes et al. 2015). Shellfish have a relatively uniform lipid/protein carbon weight composition with wtC\% of c. $90 \pm 2.5 \%$ and $10 \pm$ $2.5 \%$, respectively (USDA database). Protein and lipid concentrations in sea mammals are also variable. Food composition databases offer a limited range of comparanda. Based on published values, sea mammal (meat and subcutaneous fat) composition values were calculated as $\mathrm{wtC} \%$ protein $=45 \pm$ $5 \%$ and $\mathrm{wtC} \%$ energy $=55 \pm 5 \%$.

\section{Food source $\delta^{13} \mathrm{C}$ and $\delta^{15} \mathrm{~N}$ values}

There were three broad categories of isotopically distinct food sources that may have contributed to the diets of post-glacial hunter-gatherers: (1) wild plant foods; (2) terrestrial wild mammals; (3) aquatic resources (freshwater and marine).

- Wild plant foods. Bayesian models of diets should elucidate the role of plant foods in LUP and Mesolithic diets. However, studies of the $\delta^{13} \mathrm{C}$ and $\delta^{15} \mathrm{~N}$ values of archaeological wild plant food remains are scant. Modern comparanda may be used as proxies in dietary models (e.g. Meadows et al. 2019). $\delta^{13} \mathrm{C}$ and $\delta^{15} \mathrm{~N}$ values of hazelnuts and mushrooms, two protein-rich plant foods from the 'primaeval' forest of Białowieża, Poland, were reported by Selva et al. (2012) to be $\delta^{13} \mathrm{C}=-31.3 \pm 0.4$ and $\delta^{15} \mathrm{~N}=-0.8 \pm 0.4$ and $\delta^{13} \mathrm{C}=-20.1 \pm 0.3$ and $\delta^{15} \mathrm{~N}=-0.2 \pm 0.7$, respectively. However, modern proxies may introduce further uncertainties into dietary reconstructions. Although these data were corrected for the Suess effect (recent ${ }^{13} \mathrm{C}$-depletion of atmospheric $\mathrm{CO}_{2}$ resulting from the use of fossil fuels (Keeling 1979)), the samples were not pre-treated to remove lipids. Lipids are generally ${ }^{13} \mathrm{C}$-depleted relative to proteins and carbohydrates (DeNiro and Epstein 1978). While $\delta^{15} \mathrm{~N}$ values reported in Selva et al. (2012) reflect the plant protein values (as lipids do not contain any nitrogen), the $\delta^{13} \mathrm{C}$ values reflect combined plant protein and energy. To accurately model whole diet, $\delta^{13} \mathrm{C}$ values for both plant protein and energy are required. Therefore, the $\delta^{13} \mathrm{C}$ and $\delta^{15} \mathrm{~N}$ values were taken from the mean $\delta^{15} \mathrm{~N}$ of hazelnuts and mushrooms of $-0.4 \pm 1.0 \%$ in Selva et al. (2012) and mean energy $\delta^{13} \mathrm{C}$ of $-23.5 \pm 1.0 \%$ and mean protein $\delta^{13} \mathrm{C}$ of $-26.0 \pm 1.0 \%$ o published in Bogaard et al. (2013).

- Terrestrial mammals. Climatic changes through the Lateglacial and Holocene are mirrored in temporal variation in environmental $\delta^{13} \mathrm{C}$ and $\delta^{15} \mathrm{~N}$ (Drucker et al. 2003; Stevens and Hedges 2004). In the following analyses, food source $\delta^{13} \mathrm{C}$ and $\delta^{15} \mathrm{~N}$ specific to the time period of each site are utilised. Typical isotope values of Lateglacial and Early-Middle Holocene terrestrial animals were determined from archaeological specimens (see Tables 1 and 2; site location, dates and sample details are provided in Table S1). For our Lateglacial models, stable isotope values of animal remains ${ }^{14} \mathrm{C}$ dated to the BøllingAllerød were used. There are multiple analyses of several of the Lateglacial animal bone samples. The values used here for palaeodietary modelling are either the most recently published measurements, or in the case of those measured in separate studies for ${ }^{14} \mathrm{C}$ determination and dietary studies, the latter values are used. Mean bone collagen $\delta^{13} \mathrm{C}$ and $\delta^{15} \mathrm{~N}$ values (with standard error of the mean, cf. Hedges et al. 2007) of Bølling-Allerød terrestrial herbivores $(n=50)$ were $-20.3 \pm 0.1 \%$ and $1.8 \pm$ $0.2 \%$, respectively. Incorporating the bone collagen to protein tissue offset and food source uncertainties, Lateglacial terrestrial herbivores have mean protein $\delta^{13} \mathrm{C}=-22.3 \pm 1.0 \%$, mean energy $\delta^{13} \mathrm{C}=-28.3 \pm$ $1.0 \%$ and mean protein $\delta^{15} \mathrm{~N}=3.8 \pm 1.0 \%$. Mean bone collagen $\delta^{13} \mathrm{C}$ and $\delta^{15} \mathrm{~N}$ values (with standard error) of Mesolithic terrestrial herbivores and omnivores $(n=36)$ were $-21.6 \pm 0.2 \%$ ond $4.0 \pm 0.4 \%$, respectively. Incorporating the bone collagen to protein tissue offset and food source uncertainties, Mesolithic terrestrial herbivores and omnivores have mean protein $\delta^{13} \mathrm{C}=-23.6 \pm$ $1.0 \%$ and $\delta^{15} \mathrm{~N}=6.0 \pm 1.0 \%$.

- Aquatic food sources can be grouped into two main isotopic categories: freshwater and marine. Marine food sources can be subdivided into three further isotopic groups: (1) shellfish, (2) fish and seabirds, and (3) sea mammals. Establishing the $\delta^{13} \mathrm{C}$ and $\delta^{15} \mathrm{~N}$ values of aquatic resources is non-trivial. Carbon and nitrogen stable isotope values of archaeological specimens of marine fish and seal are available for the Mesolithic and later Holocene periods (see Table 3). However, no data for archaeological freshwater resources or marine shellfish are available for Great Britain. Therefore, stable isotope values of archaeological freshwater fish are drawn from continental European sites (see Table 3), while shellfish flesh values are derived from modern comparanda (Bownes 2018). Mean carbon and nitrogen stable isotope values of archaeological marine fish are $\delta^{13} \mathrm{C}=-12.9 \pm 0.1 \%$ and $\delta^{15} \mathrm{~N}=14.0 \pm 0.1 \%$ o $(n=28)$. Mean carbon and nitrogen stable isotope 
Table $1 \quad \delta^{13} \mathrm{C}$ and $\delta^{15} \mathrm{~N}$ values of Lateglacial (first half of the Bølling-Allerød Interstadial) herbivores used in FRUITS models

\begin{tabular}{|c|c|c|c|c|c|c|}
\hline Site name & Laboratory ID & Species & $\delta^{13} \mathrm{C} \%$ & $\delta^{15} \mathbf{N} \%$ & $\mathbf{C} / \mathbf{N}$ & Reference \\
\hline Aveline's Hole & OxA-18075 & Rangifer tarandus & -19.0 & 2.6 & 3.3 & Stevens et al. 2008; Stevens et al. 2010 \\
\hline Aveline's Hole & OxA-1121 & Cervus elaphus & -20.1 & 2.1 & 3.2 & Hedges et al. 1987; Stevens et al. 2008 \\
\hline Aveline's Hole & OxA-17722 & Cervus elaphus & -20.1 & 2.9 & 3.2 & Jacobi and Higham 2009 \\
\hline Brown Bank & GrA-28364 & Bos/Bison & -20.6 & 3.9 & 3.1 & Amkreutz et al. 2018 \\
\hline Dead Man's Cave & OxA-6327 & Equus ferus & -20.3 & 0.7 & 3.4 & Stevens and Hedges 2004 \\
\hline Foxhole & OxA-25146 & Rangifer tarandus & -19.7 & 2.7 & 3.2 & Schulting et al. 2013 \\
\hline Gough's Old Cave & OxA-17834 & Equus ferus & -20.1 & 0.7 & 3.2 & Gowlett et al. 1986; Richards et al. 2000 \\
\hline Gough's Cave & OxA-588 & Bos & -19.4 & 2.8 & 3.1 & Gowlett et al. 1986; Richards et al. 2000 \\
\hline Gough's Cave & OxA-813 & Bos primigenius & -19.8 & 2.8 & 3.2 & Gowlett et al. 1986; Stevens et al. 2010 \\
\hline Gough's Cave & OxA-16378 & Cervus elaphus & -19.8 & 3.2 & 3.2 & Jacobi and Higham 2009 \\
\hline Gough's Cave & OxA-466 & Cervus elaphus & -19.5 & 2.7 & 3.1 & Gillespie et al. 1985; Richards et al. 2000 \\
\hline Gough's Cave & OxA-17845 & Cervus elaphus & -19.6 & 2.8 & 3.2 & Jacobi and Higham 2009 \\
\hline Gough's Cave & OxA-1071 & Cervus elaphus & -19.6 & 2.4 & 3.2 & Hedges et al. 1987; Stevens et al. 2010 \\
\hline Gough's Cave & OxA-17833 & Equus ferus & -20.7 & 1.1 & 3.2 & Jacobi and Higham 2009 \\
\hline Gough's Cave & OxA-465 & Equus ferus & -19.9 & 0.7 & 3.1 & Gillespie et al. 1985: Richards et al. 2000 \\
\hline Gough's Cave & OxA-17832 & Equus ferus & -20.9 & 1.5 & 3.2 & Jacobi and Higham 2009 \\
\hline Gough's Cave & OxA-464 & Equus ferus & -20.2 & 1.2 & 3.3 & Gowlett et al. 1986; Stevens et al. 2010 \\
\hline Gough's Cave & OxA-3413 & Equus ferus & -20.3 & 0.4 & 3.2 & Hedges et al. 1994; Stevens et al. 2010 \\
\hline Gough's Cave & OxA-18064 & Rangifer tarandus & -19.2 & 1.8 & 3.2 & Jacobi and Higham 2009 \\
\hline Gough's Cave & OxA-4106 & Equus ferus & -20.1 & 3.1 & 3.2 & Hedges et al. 1994; Stevens et al. 2010 \\
\hline Gough's Cave & OxA-18068 & Equus ferus & -20.1 & 3.1 & 3.2 & Jacobi and Higham 2009 \\
\hline Gough's Cave & OxA-18065 & Equus ferus & -20.5 & 1.6 & 3.2 & Jacobi and Higham 2009 \\
\hline Gough's Cave & OxA-3452 & Equus ferus & -20.8 & 1.4 & 3.3 & Hedges et al. 1994; Stevens et al. 2010 \\
\hline Gough's Cave & OxA-12104 & Equus ferus & -20.6 & 1.0 & 3.1 & Jacobi and Higham 2009 \\
\hline Gough's Cave & OxA-11241 & Equus ferus & -20.8 & 0.7 & 3.3 & Stevens and Hedges 2004 \\
\hline Gough's Cave & OxA-18067 & Cervus elaphus & -20.2 & 2.6 & 3.2 & Jacobi and Higham 2009 \\
\hline Gough's Cave & OxA-16292 & Equus ferus & -20.5 & 0.4 & 3.2 & Jacobi and Higham 2009 \\
\hline Gough's Cave & OxA-17846 & Mammuthus & -21.2 & 6.8 & 3.2 & Jacobi and Higham 2009 \\
\hline Gough's Cave & OxA-18035 & Herbivore & -20.2 & 0.1 & 3.2 & Jacobi and Higham 2009 \\
\hline Kendrick's Cave & OxA-6146 & Bos/Bison & -20.5 & 2.8 & 3.3 & Richards et al. 2005; Stevens et al. 2010 \\
\hline Kendrick's Cave & OxA-6116 & Capreolus capreolus & -21.7 & 3.1 & 3.3 & Jacobi and Higham 2009 \\
\hline Kent's Cavern & OxA-17544 & Bovid & -19.3 & 4.6 & 3.3 & Jacobi and Higham 2009: Stevens and Hedges 2004 \\
\hline Kent's Cavern & OxA-6669 & Equus ferus & -20.8 & 1.0 & 3.3 & Stevens and Hedges 2004; Stevens et al. 2010 \\
\hline Kent's Cavern & OxA-17723 & Equus ferus & -20.4 & 1.7 & 3.2 & Jacobi and Higham 2009: Stevens and Hedges 2004 \\
\hline Kent's Cavern & OxA-17545 & Equus ferus & -20.0 & 1.5 & 3.3 & Jacobi and Higham 2009: Stevens et al. 2010 \\
\hline King Arthur's Cave & OxA-17725 & Equus ferus & -20.4 & 0.3 & 3.2 & Jacobi and Higham 2009 \\
\hline King Arthur's Cave & OxA-6631 & Equus ferus & -20.7 & 1.4 & 3.3 & Stevens and Hedges 2004 \\
\hline King Arthur's Cave & OxA-6733 & Equus ferus & -20.7 & 0.2 & 3.3 & Stevens and Hedges 2004 \\
\hline King Arthur's Cave & OxA-6732 & Equus ferus & -20.4 & 1.3 & 3.3 & Stevens and Hedges 2004 \\
\hline Mother Grundy's Parlour & OxA-6666 & Equus ferus & -21.0 & 1.0 & 3.3 & Stevens and Hedges 2004 \\
\hline Mother Grundy's Parlour & OxA-3398 & Equus ferus & -20.6 & 0.7 & 3.3 & Stevens and Hedges 2004 \\
\hline Mother Grundy's Parlour & OxA-3400 & Equus ferus & -20.7 & 0.7 & 3.3 & Stevens and Hedges 2004 \\
\hline Mother Grundy's Parlour & OxA-8738 & Equus ferus & -20.6 & 1.4 & 3.3 & Stevens and Hedges 2004 \\
\hline Mother Grundy's Parlour & OxA-8739 & Equus ferus & -19.6 & 1.7 & 3.4 & Stevens and Hedges 2004 \\
\hline Pixies' Hole & OxA-14068 & Equus ferus & -20.2 & -0.2 & 3.3 & Jacobi and Higham 2009 \\
\hline Robin Hood's Cave & OxA-6324 & Equus ferus & -20.6 & 1.8 & 3.3 & Stevens and Hedges 2004 \\
\hline Sun Hole & OxA-14438 & Equus ferus & -20.4 & 1.1 & 3.2 & Jacobi and Higham 2009 \\
\hline Sun Hole & OxA-14476a & Equus ferus & -20.7 & 0.4 & 3.4 & Jacobi and Higham 2009 \\
\hline Sun Hole & OxA-14477a & Equus ferus & -20.7 & 1.1 & 3.5 & Jacobi and Higham 2009 \\
\hline Sun Hole & OxA-18705 & Equus ferus & -20.1 & 1.8 & 3.2 & Jacobi and Higham 2009 \\
\hline
\end{tabular}

values of Lateglacial fish from freshwater systems are $\delta^{13} \mathrm{C}=-21.6 \pm 1.0 \%$ and $\delta^{15} \mathrm{~N}=9.0 \pm 0.6 \%$ o $(n=7)$. Mean carbon and nitrogen stable isotope values of Mesolithic fish from freshwater systems are $\delta^{13} \mathrm{C}=$ $-20.6 \pm 0.7 \%$ and $\delta^{15} \mathrm{~N}=9.4 \pm 0.2 \%$ o $(n=39)$. The $\delta^{13} \mathrm{C}$ and $\delta^{15} \mathrm{~N}$ values for the Lateglacial and Mesolithic fish are not statistically different (Mann-
Whitney $U$ test, $\delta^{13} \mathrm{C} p=0.614 ; \delta^{15} \mathrm{~N} p=0.392$ ), and so have been grouped for dietary modelling. Mean values for archaeological seal are $\delta^{13} \mathrm{C}=-12.3 \pm$ $0.3 \%$ and $\delta^{15} \mathrm{~N}=18.2 \pm 0.3 \%$ o $(n=9)$. Incorporating the bone collagen to protein tissue offset and food source uncertainties, the marine fish protein values are $\delta^{13} \mathrm{C}=-13.9 \pm 1.0 \%$ and $\delta^{15} \mathrm{~N}=16.0 \pm 1.0 \%$; 
Table $2 \quad \delta^{13} \mathrm{C}$ and $\delta^{15} \mathrm{~N}$ values of Mesolithic fauna used in FRUITS models

\begin{tabular}{|c|c|c|c|c|c|c|}
\hline Site name & Laboratory ID & Species & $\delta^{13} \mathrm{C} \%$ & $\delta^{15} \mathbf{N} \%$ & $\mathbf{C} / \mathbf{N}$ & Reference \\
\hline \multicolumn{7}{|l|}{ Omnivores } \\
\hline Cnoc Coig & OxA-29937 & Sus & -21.2 & 4.3 & 3.4 & Charlton et al. 2016 \\
\hline Cnoc Coig & OxA-29936 & Sus & -21.0 & 4.6 & 3.3 & Charlton et al. 2016 \\
\hline Cnoc Coig & & Sus & -18.8 & 10.2 & 3.4 & Charlton et al. 2016 \\
\hline Eel Point & & Sus scrofa & -18.5 & 8.6 & 3.3 & Schulting and Richards 2002a \\
\hline Little Hoyle Cave & & Sus sp. (domestic?) & -21.2 & 7.2 & 3.4 & Schulting and Richards 2002a \\
\hline Risga & GUsi3495 & Sus & -22.3 & 2.2 & 3.4 & Bownes 2018 \\
\hline Risga & GUsi3496 & Sus & -21.2 & 5.0 & 3.4 & Bownes 2018 \\
\hline Star Carr & & Sus scrofa & -21.9 & 4.4 & 3.3 & Schulting and Richards 2009 \\
\hline \multicolumn{7}{|l|}{ Herbivores } \\
\hline Eel Point & & Cervus elaphus & -20.5 & 4.4 & 3.3 & Schulting and Richards 2002a \\
\hline Eel Point & & Cervus elaphus & -21.8 & 5.4 & 3.3 & Schulting and Richards 2002a \\
\hline Little Hoyle Cave & & Bos sp. & -22.2 & 5.6 & 3.2 & Schulting and Richards 2002a \\
\hline Little Hoyle Cave & & Bos sp. & -22.2 & 6.3 & 3.4 & Schulting and Richards 2002a \\
\hline Nanna's Cave & & Bos sp. & -21.2 & 7.3 & 3.2 & Schulting and Richards 2002a \\
\hline Ogof-yr-Ychen & & Cervus elaphus & -22.3 & 2.4 & 3.1 & Schulting and Richards 2002a \\
\hline Potter's Cave & & Canis sp. & -19.8 & 8.0 & 3.4 & Schulting and Richards 2002a \\
\hline Potter's Cave & & Canis sp. & -20.4 & 8.4 & 3.1 & Schulting and Richards 2002a \\
\hline Raschoille Cave & OxA-8396 & Cervus elaphus & -21.8 & 2.9 & 3.3 & This study \\
\hline Raschoille Cave & OxA-8397 & Cervus elaphus & -21.5 & 2.8 & 3.3 & This study \\
\hline Raschoille Cave & OxA-8398 & Cervus elaphus & -21.6 & 2.6 & 3.2 & This study \\
\hline Raschoille Cave & OxA-8535 & Cervus elaphus & -21.4 & 0.6 & 3.5 & This study \\
\hline Risga & GUsi3485 & Capreolus capreolus & -21.8 & 2.0 & 3.2 & Bownes 2018 \\
\hline Risga & GUsi3487 & Cervus elaphus & -21.8 & 2.1 & 3.3 & Bownes 2018 \\
\hline Risga & GUsi3488 & Cervus elaphus & -22.1 & 2.4 & 3.3 & Bownes 2018 \\
\hline Risga & GUsi3489 & Cervus elaphus & -22.1 & 2.5 & 3.3 & Bownes 2018 \\
\hline Risga & GUsi3491 & Cervus elaphus & -21.9 & 1.3 & 3.4 & Bownes 2018 \\
\hline Risga & GUsi3492 & Cervus elaphus & -22.5 & 2.7 & 3.3 & Bownes 2018 \\
\hline Risga & GUsi3493 & Cervus elaphus & -22.0 & 2.6 & 3.4 & Bownes 2018 \\
\hline Star Carr & & Bos primigenius & -21.5 & 3.3 & 3.2 & Schulting and Richards 2009 \\
\hline Star Carr & & Alces alces & -21.6 & 2.7 & 3.3 & Schulting and Richards 2009 \\
\hline Star Carr & & Alces alces & -21.5 & 2.0 & 3.2 & Schulting and Richards 2009 \\
\hline Star Carr & & Capreolus capreolus & -23.1 & 3.2 & 3.3 & Schulting and Richards 2009 \\
\hline Star Carr & & Cervus elaphus & -22.6 & 4.0 & 3.3 & Schulting and Richards 2009 \\
\hline Ulva Cave & GUsi3748 & Cervus elaphus & -24.5 & 4.9 & 3.3 & Bownes 2018 \\
\hline Ulva Cave & GUsi3852 & Cervus elaphus & -21.8 & 0.8 & 3.3 & Bownes 2018 \\
\hline Ulva Cave & GUsi3853 & Cervus elaphus & -22.2 & 3.8 & 3.3 & Bownes 2018 \\
\hline Ulva Cave & GUsi3855 & Cervus elaphus & -21.6 & 1.6 & 3.4 & Bownes 2018 \\
\hline
\end{tabular}

freshwater fish values are $\delta^{13} \mathrm{C}=-21.7 \pm 1.0 \%$ and $\delta^{15} \mathrm{~N}=11.4 \pm 1.0 \%$; while seal protein values are $\delta^{13} \mathrm{C}=-14.6 \pm 1.0 \% \circ$ and $\delta^{15} \mathrm{~N}=17.6 \pm 1.0 \%$.

$\delta^{13} \mathrm{C}$ and $\delta^{15} \mathrm{~N}$ of modern shellfish flesh are used in dietary models (e.g. Bonsall et al. 2009; Montgomery et al. 2013). Although such data should be corrected for the Suess effect, the impact on oceanic $\delta^{13} \mathrm{C}$ has been smaller than on atmospheric $\delta^{13} \mathrm{C}$. In the North Atlantic, ${ }^{13} \mathrm{C}$-depletion of up to $0.8 \%$ is evident (Eide et al. 2017). Lipid extraction has generally been undertaken before measuring the $\delta^{13} \mathrm{C}$ and $\delta^{15} \mathrm{~N}$ signals of shellfish protein. However, lipid extraction has been demonstrated to alter $\delta^{15} \mathrm{~N}$ values in a non-predictable manner (e.g. Post et al. 2007; Logan et al. 2008). It is best practice to use the $\delta^{13} \mathrm{C}$ values from a lipid-extracted sample alongside 
Table $3 \quad \delta^{13} \mathrm{C}$ and $\delta^{15} \mathrm{~N}$ values of aquatic food sources used in FRUITS models

\begin{tabular}{|c|c|c|c|c|c|c|c|c|}
\hline Site name & Laboratory ID & Location & Date & Species & $\delta^{13} \mathrm{C} \%$ & $\delta^{15} \mathbf{N} \%$ & $\mathrm{C} / \mathrm{N}$ & Reference \\
\hline \multicolumn{9}{|l|}{ Freshwater fish } \\
\hline Pont d'Ambon & PAM6000 & Southwest France & Mesolithic & Esox lucius & -22.2 & 9.5 & 3.1 & $\begin{array}{l}\text { Drucker and } \\
\text { Bocherens } 2004\end{array}$ \\
\hline Pont d'Ambon & PAM5900 & Southwest France & Mesolithic & Cyprinidae & -21.5 & 9.4 & 3.1 & $\begin{array}{l}\text { Drucker and } \\
\text { Bocherens } 2004\end{array}$ \\
\hline Pont d'Ambon & PAM6200 & Southwest France & Mesolithic & $\begin{array}{l}\text { Anguilla } \\
\text { anguilla }\end{array}$ & -23.7 & 8.0 & 3.0 & $\begin{array}{l}\text { Drucker and } \\
\text { Bocherens } 2004\end{array}$ \\
\hline Daḅki 9 & D9P. $2 a+b$ & W Pomerania, Poland & $\begin{array}{l}\text { Mesolithic/Early } \\
\text { Neolithic }\end{array}$ & Esox lucius & -24.0 & 10.4 & 3.5 & Robson et al. 2016 \\
\hline Daḅki 9 & D9P. $4 a+b$ & W Pomerania, Poland & $\begin{array}{l}\text { Mesolithic/Early } \\
\text { Neolithic }\end{array}$ & Esox lucius & -21.8 & 10.4 & 3.3 & Robson et al. 2016 \\
\hline Daḅki 9 & D9P.5a, b + c & W Pomerania, Poland & $\begin{array}{l}\text { Mesolithic/Early } \\
\text { Neolithic }\end{array}$ & Esox lucius & -24.2 & 8.6 & 3.5 & Robson et al. 2016 \\
\hline Daḅki 9 & D9PF.4a & W Pomerania, Poland & $\begin{array}{l}\text { Mesolithic/Early } \\
\text { Neolithic }\end{array}$ & Perca fluviatilis & -18.8 & 9.8 & 3.6 & Robson et al. 2016 \\
\hline Daḅki 9 & D9PF.6a $+b$ & W Pomerania, Poland & $\begin{array}{l}\text { Mesolithic/Early } \\
\text { Neolithic }\end{array}$ & Perca fluviatilis & -17.9 & 9.1 & 3.3 & Robson et al. 2016 \\
\hline Daḅki 9 & D9Z.1a+b & W Pomerania, Poland & $\begin{array}{l}\text { Mesolithic/Early } \\
\text { Neolithic }\end{array}$ & $\begin{array}{l}\text { Sander } \\
\quad \text { lucioperca }\end{array}$ & -24.5 & 9.9 & 3.3 & Robson et al. 2016 \\
\hline Daḅki 9 & D9Z. $2 a+b$ & W Pomerania, Poland & $\begin{array}{l}\text { Mesolithic/Early } \\
\text { Neolithic }\end{array}$ & $\begin{array}{l}\text { Sander } \\
\quad \text { lucioperca }\end{array}$ & -21.6 & 11.8 & 3.3 & Robson et al. 2016 \\
\hline Daḅki 9 & D9Z.6a + b & W Pomerania, Poland & $\begin{array}{l}\text { Mesolithic/Early } \\
\text { Neolithic }\end{array}$ & $\begin{array}{l}\text { Sander } \\
\quad \text { lucioperca }\end{array}$ & -21.1 & 11.9 & 3.4 & Robson et al. 2016 \\
\hline Holmegard I & AAR8854/1922c & Denmark & Mesolithic & Esox lucius & -15.4 & 7.8 & 3.6 & Fischer et al. 2007 \\
\hline Holmegard IV & 1944-38D & Denmark & Mesolithic & Esox lucius & -22.8 & 10.0 & 3.6 & Fischer et al. 2007 \\
\hline Mullerup & ВCH198:21a + b & Denmark & Mesolithic & Esox lucius & -9.3 & 9.4 & 3.6 & Fischer et al. 2007 \\
\hline Mullerup & 5/ACQ59:19+40 & Denmark & Mesolithic & Esox lucius & -8.0 & 9.2 & 3.4 & Fischer et al. 2007 \\
\hline Mullerup & 9/ACQ66a:24 + 43 & Denmark & Mesolithic & Esox lucius & -9.5 & 8.7 & 3.3 & Fischer et al. 2007 \\
\hline Storelyng VI & AF9093 & Denmark & $\begin{array}{l}\text { Middle/Late } \\
\text { Mesolithic }\end{array}$ & Esox lucius & -24.0 & 7.8 & 3.2 & Fischer et al. 2007 \\
\hline Storelyng VI & AF9440 & Denmark & $\begin{array}{l}\text { Middle/Late } \\
\text { Mesolithic }\end{array}$ & Esox lucius & -25.9 & 6.6 & 3.4 & Fischer et al. 2007 \\
\hline Argus & AAR-8605 & Denmark & Middle Mesolithic & Esox lucius & -13.3 & 11.8 & 3.5 & Fischer et al. 2007 \\
\hline Noyen-sur-Seine & NO7600 & Northern France & Mesolithic & $\begin{array}{l}\text { Anguilla } \\
\text { anguilla }\end{array}$ & -23.8 & 8.3 & 3.3 & $\begin{array}{l}\text { Bocherens et al. 2007; } \\
\text { Drucker et al. } 2016\end{array}$ \\
\hline Abri du Pape & BP16 & France & Mesolithic & Esox lucius & -22.5 & 10.9 & 3.3 & Drucker et al. 2016 \\
\hline Abri du Pape & BP20 & France & Mesolithic & Cyprinidae & -21.2 & 8.0 & 3.2 & Drucker et al. 2016 \\
\hline Abri du Pape & BP21 & France & Mesolithic & Cyprinidae & -22.3 & 9.5 & 3.2 & Drucker et al. 2016 \\
\hline Trou de Chaleux & VERT-84/85 & France & Mesolithic & Salmo trutta & -18.7 & 8.7 & 3.5 & Drucker et al. 2018 \\
\hline Trou du Sureau & VERT-72/74 & France & Mesolithic & Lota lota & -23.2 & 6.6 & 3.2 & Drucker et al. 2018 \\
\hline Trou du Frontal & VERT-91/94 & France & Mesolithic & Lota lota & -21.8 & 10.5 & 3.1 & Drucker et al. 2018 \\
\hline Bois Laiterie & BP 3/11 & France & Mesolithic & Lota lota & -24.1 & 9.8 & 3.1 & Drucker et al. 2018 \\
\hline Bois Laiterie & BP 8 & France & Mesolithic & Lota lota & -23.8 & 7.7 & 3.1 & Drucker et al. 2018 \\
\hline Bois Laiterie & BP 5 & France & Mesolithic & Salmo trutta & -20.5 & 9.2 & 3.3 & Drucker et al. 2018 \\
\hline Bois Laiterie & BP 6/7/15 & France & Mesolithic & Salmo trutta & -19.8 & 9.4 & 3.3 & Drucker et al. 2018 \\
\hline Bois Laiterie & BP 13 & France & Mesolithic & Salmo trutta & -20.5 & 8.9 & 3.2 & Drucker et al. 2018 \\
\hline Bois Laiterie & BP 14 & France & Mesolithic & Salmo trutta & -19.8 & 8.7 & 3.2 & Drucker et al. 2018 \\
\hline Trou du Frontal & VERT 110 & France & Mesolithic & Esox lucius & -20.5 & 10.6 & 3.4 & Drucker et al. 2018 \\
\hline Trou du Frontal & VERT 111 & France & Mesolithic & Esox lucius & -23.7 & 8.1 & 3.3 & Drucker et al. 2018 \\
\hline Trou de Chaleux & VERT 90 & France & Mesolithic & Esox lucius & -21.7 & 10.3 & 3.3 & Drucker et al. 2018 \\
\hline Trou de Chaleux & VERT 82 & France & Mesolithic & $\begin{array}{l}\text { Chondrostoma } \\
\text { nasus }\end{array}$ & -20.1 & 8.7 & 3.1 & Drucker et al. 2018 \\
\hline Trou du Frontal & VERT 112 & France & Mesolithic & Cyprinidae & -21.9 & 11.7 & 3.2 & Drucker et al. 2018 \\
\hline Trou du Frontal & VERT 113 & France & Mesolithic & Cyprinidae & -21.7 & 10.6 & 3.2 & Drucker et al. 2018 \\
\hline Trou du Frontal & VERT 114 & France & Mesolithic & Cyprinidae & -21.8 & 10.5 & 3.4 & Drucker et al. 2018 \\
\hline Šandalja II & S-EVA-5255 & Istria, Croatia & Lateglacial & Esox luscius & -23.7 & 8.5 & 3.4 & Richards et al. 2015 \\
\hline Šandalja II & S-EVA-5254 & Istria, Croatia & Lateglacial & Esox luscius & -22.7 & 9.3 & 3.4 & Richards et al. 2015 \\
\hline Šandalja II & S-EVA-5253 & Istria, Croatia & Lateglacial & Esox luscius & -22.6 & 9.2 & 3.5 & Richards et al. 2015 \\
\hline Šandalja II & S-EVA-5252 & Istria, Croatia & Lateglacial & Esox luscius & -24.1 & 9.1 & 3.5 & Richards et al. 2015 \\
\hline Pont d'Ambon & PAM6400 & Southwest France & Lateglacial & $\begin{array}{l}\text { Anguilla } \\
\quad \text { anguilla }\end{array}$ & -20.8 & 8.5 & 3.1 & $\begin{array}{l}\text { Drucker and } \\
\text { Bocherens } 2004\end{array}$ \\
\hline
\end{tabular}


Table 3 (continued)

\begin{tabular}{|c|c|c|c|c|c|c|c|c|}
\hline Site name & Laboratory ID & Location & Date & Species & $\delta^{13} \mathrm{C} \%$ & $\delta^{15} \mathbf{N} \%$ & $\mathbf{C} / \mathbf{N}$ & Reference \\
\hline Pont d'Ambon & PAM6600 & Southwest France & Lateglacial & Salmonidae & -16.1 & 12.1 & 2.9 & $\begin{array}{l}\text { Drucker and } \\
\text { Bocherens } 2004\end{array}$ \\
\hline Pont d'Ambon & PAM6300 & Southwest France & Lateglacial & Cyprinidae & -21.1 & 6.6 & 3.1 & $\begin{array}{l}\text { Drucker and } \\
\text { Bocherens } 2004\end{array}$ \\
\hline \multicolumn{9}{|l|}{ Marine fish } \\
\hline An Corran & & Skye & Neolithic & Gadus morhua & -13.6 & 15.3 & & Milner and Craig 2012 \\
\hline Bornish & 703 & $\begin{array}{l}\text { South Uist, Outer } \\
\text { Hebrides }\end{array}$ & $\begin{array}{c}\text { 12th-thirteenth } \\
\text { century } \mathrm{AD}\end{array}$ & Gadus morhua & -12.9 & 14.5 & 3.3 & Barrett et al. 2011 \\
\hline Bornish & 706 & $\begin{array}{l}\text { South Uist, Outer } \\
\text { Hebrides }\end{array}$ & $\begin{array}{l}\text { Thirteenth century } \\
\text { AD }\end{array}$ & Gadus morhua & -11.3 & 15.4 & 3.3 & Barrett et al. 2011 \\
\hline Bornish & 708 & $\begin{array}{l}\text { South Uist, Outer } \\
\text { Hebrides }\end{array}$ & $\begin{array}{l}\text { Thirteenth century } \\
\text { AD }\end{array}$ & Gadus morhua & -13.1 & 13.8 & 3.3 & Barrett et al. 2011 \\
\hline Bornish & 713 & $\begin{array}{l}\text { South Uist, Outer } \\
\text { Hebrides }\end{array}$ & $\begin{array}{l}\text { 12th-thirteenth } \\
\text { century } \mathrm{AD}\end{array}$ & Gadus morhua & -13.2 & 13.8 & 3.3 & Barrett et al. 2011 \\
\hline Know of Skea & 205 & Westray, Orkney & $\begin{array}{c}\text { 15th-sixteenth } \\
\text { century } \mathrm{AD}\end{array}$ & Gadus morhua & -13.4 & 14.9 & 3.6 & Barrett et al. 2011 \\
\hline Know of Skea & 208 & Westray, Orkney & $\begin{array}{c}\text { 15th-sixteenth } \\
\text { century AD }\end{array}$ & Gadus morhua & -13.4 & 14.2 & 3.5 & Barrett et al. 2011 \\
\hline Know of Skea & 209 & Westray, Orkney & $\begin{array}{c}\text { 15th-sixteenth } \\
\text { century AD }\end{array}$ & Gadus morhua & -13.5 & 14.6 & 3.6 & Barrett et al. 2011 \\
\hline Quoygrew & 7 & Westray, Orkney & Twelfth century AD & Gadus morhua & -12.5 & 13.7 & 3.3 & Barrett et al. 2011 \\
\hline Quoygrew & 10 & Westray, Orkney & Twelfth century AD & Gadus morhua & -13.4 & 13.7 & 3.3 & Barrett et al. 2011 \\
\hline Quoygrew & 11 & Westray, Orkney & Twelfth century AD & Gadus morhua & -13.6 & 13.7 & 3.3 & Barrett et al. 2011 \\
\hline Quoygrew & 22 & Westray, Orkney & Twelfth century AD & Gadus morhua & -12.6 & 13.5 & 3.2 & Barrett et al. 2011 \\
\hline Quoygrew & 25 & Westray, Orkney & Twelfth century AD & Gadus morhua & -12.9 & 14.5 & 3.4 & Barrett et al. 2011 \\
\hline Quoygrew & 26 & Westray, Orkney & Twelfth century AD & Gadus morhua & -13.7 & 14.4 & 3.4 & Barrett et al. 2011 \\
\hline Quoygrew & 28 & Westray, Orkney & Twelfth century AD & Gadus morhua & -13.4 & 14.7 & 3.4 & Barrett et al. 2011 \\
\hline Quoygrew & 50 & Westray, Orkney & Twelfth century AD & Gadus morhua & -12.4 & 11.9 & 3.3 & Barrett et al. 2011 \\
\hline Quoygrew & 73 & Westray, Orkney & Twelfth century AD & Gadus morhua & -12.5 & 14.9 & 3.2 & Barrett et al. 2011 \\
\hline Quoygrew & 100 & Westray, Orkney & Twelfth century AD & Gadus morhua & -11.9 & 14.7 & 3.2 & Barrett et al. 2011 \\
\hline Quoygrew & 102 & Westray, Orkney & Twelfth century AD & Gadus morhua & -12.4 & 14.8 & 3.2 & Barrett et al. 2011 \\
\hline Quoygrew & 106 & Westray, Orkney & Twelfth century AD & Gadus morhua & -13.1 & 13.5 & 3.3 & Barrett et al. 2011 \\
\hline Quoygrew & 116 & Westray, Orkney & Twelfth century AD & Gadus morhua & -13.5 & 14.3 & 3.5 & Barrett et al. 2011 \\
\hline Quoygrew & 145 & Westray, Orkney & Twelfth century AD & Gadus morhua & -13.4 & 13.9 & 3.3 & Barrett et al. 2011 \\
\hline Quoygrew & 582 & Westray, Orkney & Fifteenth century AD & Gadus morhua & -12.2 & 13.8 & 3.2 & Barrett et al. 2011 \\
\hline Quoygrew & 583 & Westray, Orkney & Fifteenth century $\mathrm{AD}$ & Gadus morhua & -11.9 & 14.4 & 3.2 & Barrett et al. 2011 \\
\hline Quoygrew & 584 & Westray, Orkney & Fifteenth century AD & Gadus morhua & -14.2 & 15.1 & 3.4 & Barrett et al. 2011 \\
\hline Quoygrew & 585 & Westray, Orkney & Fifteenth century $\mathrm{AD}$ & Gadus morhua & -12.3 & 14.2 & 3.3 & Barrett et al. 2011 \\
\hline Quoygrew & 587 & Westray, Orkney & Fifteenth century $\mathrm{AD}$ & Gadus morhua & -12.0 & 14.7 & 3.1 & Barrett et al. 2011 \\
\hline Potter's Cave & & $\begin{array}{l}\text { Caldey Island, } \\
\text { Pembrokeshire }\end{array}$ & Mesolithic/Neolithic & $\begin{array}{l}\text { Lophius } \\
\quad \text { piscatorius }\end{array}$ & -12.6 & 7.6 & 3.0 & $\begin{array}{l}\text { Schulting and } \\
\text { Richards 2002a }\end{array}$ \\
\hline \multicolumn{9}{|l|}{ Shellfish } \\
\hline Airds Bay & GUsi3201/3208 & Appin, Argyll & & Patellidae flesh & -14.1 & 6.3 & & Bownes 2018 \\
\hline Airds Bay & GUsi3202/3209 & Appin, Argyll & & Patellidae flesh & -15.0 & 6.7 & & Bownes 2018 \\
\hline Airds Bay & GUsi3204/3211 & Appin, Argyll & & Patellidae flesh & -15.1 & 6.3 & & Bownes 2018 \\
\hline Airds Bay & GUsi3205/3212 & Appin, Argyll & & Patellidae flesh & -15.0 & 7.1 & & Bownes 2018 \\
\hline Airds Bay & GUsi3206/3213 & Appin, Argyll & & Patellidae flesh & -14.0 & 7.0 & & Bownes 2018 \\
\hline Airds Bay & GUsi3207/3214 & Appin, Argyll & & Patellidae flesh & -15.2 & 6.7 & & Bownes 2018 \\
\hline SAMS & GUsi3215/3221 & Oban, Argyll & & Patellidae flesh & -13.6 & 7.8 & & Bownes 2018 \\
\hline SAMS & GUsi3216/3222 & Oban, Argyll & & Patellidae flesh & -15.5 & 6.9 & & Bownes 2018 \\
\hline SAMS & GUsi3217/3223 & Oban, Argyll & & Patellidae flesh & -14.7 & 6.2 & & Bownes 2018 \\
\hline SAMS & GUsi3446/3598 & Oban, Argyll & & $\begin{array}{l}\text { Littorinidae } \\
\text { flesh }\end{array}$ & -15.3 & 11.7 & & Bownes 2018 \\
\hline SAMS & GUsi3451/3603 & Oban, Argyll & & $\begin{array}{l}\text { Littorinidae } \\
\text { flesh }\end{array}$ & -13.3 & 8.5 & & Bownes 2018 \\
\hline \multicolumn{9}{|l|}{ SEAL } \\
\hline Cnoc Coig & & Oronsay & Meso? & Phocidae & -11.6 & 18.8 & 3.3 & Charlton et al. 2016 \\
\hline Cnoc Coig & & Oronsay & Meso? & Phocidae & -11.8 & 19.5 & 3.5 & Charlton et al. 2016 \\
\hline $\begin{array}{r}\text { Caisteal nan } \\
\text { Gillean II }\end{array}$ & & Scotland & Meso? & $\begin{array}{c}\text { Halichoerus } \\
\text { grypus }\end{array}$ & -11.9 & 19.1 & 3.2 & $\begin{array}{l}\text { Richards and } \\
\text { Mellars } 1998\end{array}$ \\
\hline West Voe & Wevo-3 & Sumburgh, Shetland & Neo & Phocidae & -11.9 & 16.8 & 3.0 & $\begin{array}{r}\text { Montgomery } \\
\text { et al. } 2013\end{array}$ \\
\hline
\end{tabular}


Table 3 (continued)

\begin{tabular}{|c|c|c|c|c|c|c|c|c|}
\hline Site name & Laboratory ID & Location & Date & Species & $\delta^{13} \mathrm{C} \%$ & $\delta^{15} \mathbf{N} \%$ & $\mathbf{C} / \mathbf{N}$ & Reference \\
\hline West Voe & Wevo-4 & Sumburgh, Shetland & $\mathrm{Neo}$ & Phocidae & -12.1 & 16.9 & 2.9 & $\begin{array}{r}\text { Montgomery } \\
\text { et al. } 2013\end{array}$ \\
\hline West Voe & Wevo-5 & Sumburgh, Shetland & $\mathrm{Neo}$ & Phocidae & -11.6 & 18.1 & 3.0 & $\begin{array}{r}\text { Montgomery } \\
\text { et al. } 2013\end{array}$ \\
\hline West Voe & Wevo-6 & Sumburgh, Shetland & $\mathrm{Neo}$ & Phocidae & -12.8 & 17.1 & 3.0 & $\begin{array}{l}\text { Montgomery et al. } \\
2013\end{array}$ \\
\hline West Voe & Wevo-7 & Sumburgh, Shetland & $\mathrm{Neo}$ & Phocidae & -13.7 & 19.1 & 3.1 & $\begin{array}{r}\text { Montgomery } \\
\text { et al. } 2013\end{array}$ \\
\hline West Voe & Wevo-8 & Sumburgh, Shetland & $\mathrm{Neo}$ & Phocidae & -13.5 & 18.5 & 3.1 & $\begin{array}{r}\text { Montgomery } \\
\text { et al. } 2013\end{array}$ \\
\hline
\end{tabular}

the $\delta^{15} \mathrm{~N}$ of the pre-treated sample (Sotiropoulos et al. 2004). The shellfish flesh isotope values used for this study were selected from data in Bownes (2018). Only those samples for which (1) whole and lipid extracted $\delta^{13} \mathrm{C}$ and $\delta^{15} \mathrm{~N}$ values were available and (2) with $\mathrm{C} / \mathrm{N}$ ratios in the range 2.9-3.6, characteristic of collagen, following lipid extraction (cf. DeNiro 1985) were included in this analysis. Suess effect corrected mean carbon and nitrogen stable isotope values of shellfish protein are $\delta^{13} \mathrm{C}=-14.6 \pm 0.1 \%$ and $\delta^{15} \mathrm{~N}=7.4 \pm$ $0.2 \%$ o $(n=11)$.

\section{Results and discussion}

The diets of 85 individuals from 21 sites (see Table S2) were modelled using FRUITS based on their bone collagen $\mathrm{C}$ - and $\mathrm{N}$-isotope signatures (see Tables 4, 5, 6 and 7). (Note-we treated each $\delta^{13} \mathrm{C}$ and $\delta^{15} \mathrm{~N}$ paired measurement as a separate individual unless it was specified in publications that duplicate measurements of the same skeletal element/individual had been measured; however, it is acknowledged that this figure represents a maximum number of individuals.) For the most part, we selected targets with direct AMS ${ }^{14} \mathrm{C}$ dates (Table S2). In the case of the human remains from Thatcham (Fig. 1, site 20), however, dating is based on associated palynological evidence (Churchill 1963). The radiocarbon dates cluster into three distinct periods: $15.0-13.6 \mathrm{ka}$ BP (first half of the Bølling-Allerød Interstadial), 9.5-9.0 ka BP (Early Holocene) and 8.3-6.2 ka BP (early Middle Holocene). Archaeologically, these correspond to phases within the 'Late Upper Palaeolithic' (LUP), 'Early Mesolithic' and 'Late Mesolithic', respectively.

Eleven of the 21 sites under consideration are located on or near (within $2 \mathrm{~km}$ ) the present-day coastline (Fig. 1). While distance to the sea would have varied in response to relative sea-level (RSL) changes and shoreline displacement during the post-glacial period, in most cases, the communities who used the sites during the Lateglacial or Early-Middle Holocene would likely have had direct access to coastal resources. A possible exception is Tilbury near the mouth of the River Thames (Fig. 1, site 21) where during the Late Mesolithic c. $8 \mathrm{ka}$ BP with RSL of c. -10 to $12 \mathrm{~m}$ (cf. Shennan et al. 2006, figure 7), the river habitat was likely freshwater rather than estuarine. Similarly, the Cannington

Table 4 Model 1: percentage protein (\% protein) contribution of humans at Lateglacial sites, modelled with FRUITS, assuming protein routing, incorporating six food sources

\begin{tabular}{|c|c|c|c|c|c|c|c|c|c|c|c|}
\hline ID & $\begin{array}{l}\text { Laboratory } \\
\text { ID }\end{array}$ & Site name & $\begin{array}{l}\text { Context/ } \\
\text { laboratory ID }\end{array}$ & $\begin{array}{l}\delta^{13} \mathrm{C} \\
\% o\end{array}$ & $\begin{array}{l}\delta^{15} \mathbf{N} \\
\% o\end{array}$ & $\begin{array}{l}\text { Wild } \\
\text { plants }\end{array}$ & $\begin{array}{l}\text { Terrestrial } \\
\text { mammals }\end{array}$ & Shellfish & $\begin{array}{l}\text { Marine } \\
\text { fish }\end{array}$ & Seal & Freshwater \\
\hline $\mathrm{GC} 1$ & OxA-17848 & Gough's Cave & $\begin{array}{l}\text { (1.1/4) conjoin } \\
\text { to frontal } \\
(\text { GC } 1987 \text { 169) }\end{array}$ & -19.3 & 7.6 & $74 \pm 19$ & $18 \pm 19$ & $2 \pm 2$ & $1 \pm 1$ & $2 \pm 2$ & $3 \pm 2$ \\
\hline GC2 & OxA-2236 & Gough's Cave & GC6; 1.1/3 & -19.1 & 5.4 & $89 \pm 6$ & $6 \pm 6$ & $2 \pm 1$ & $1 \pm 1$ & $1 \pm 1$ & $1 \pm 1$ \\
\hline GC3 & OxA-17847 & Gough's Cave & GC M23.1/2 & -19.0 & 7.9 & $56 \pm 27$ & $35 \pm 27$ & $2 \pm 2$ & $1 \pm 1$ & $2 \pm 2$ & $3 \pm 3$ \\
\hline GC4 & OxA-17849 & Gough's Cave & GC 1987190 & -19.3 & 7.7 & $67 \pm 25$ & $25 \pm 25$ & $2 \pm 2$ & $1 \pm 1$ & $2 \pm 2$ & $3 \pm 3$ \\
\hline $\mathrm{KC} 1$ & OxA-7003 & Kendrick's Cave & 57 & -17.9 & 13.8 & $26 \pm 20$ & $31 \pm 17$ & $3 \pm 3$ & $4 \pm 3$ & $6 \pm 5$ & $30 \pm 14$ \\
\hline $\mathrm{KC} 2$ & OxA-7004 & Kendrick's Cave & 59 & -18.0 & 13.4 & $29 \pm 21$ & $33 \pm 19$ & $3 \pm 3$ & $4 \pm 3$ & $6 \pm 5$ & $25 \pm 13$ \\
\hline $\mathrm{KC} 3$ & OxA-6114 & Kendrick's Cave & 60 & -17.7 & 13.9 & $26 \pm 18$ & $30 \pm 16$ & $4 \pm 3$ & $4 \pm 3$ & $6 \pm 5$ & $31 \pm 15$ \\
\hline $\mathrm{KC} 4$ & OxA-7002 & Kendrick's Cave & 69 & -18.1 & 13.7 & $26 \pm 20$ & $32 \pm 18$ & $3 \pm 3$ & $4 \pm 3$ & $6 \pm 5$ & $29 \pm 15$ \\
\hline $\mathrm{KC} 5$ & OxA-17089 & Kendrick's Cave & 74 & -17.7 & 13.2 & $26 \pm 20$ & $37 \pm 18$ & $4 \pm 3$ & $4 \pm 4$ & $6 \pm 5$ & $23 \pm 13$ \\
\hline SH1 & OxA-19557 & Sun Hole & M5.13/24 & -18.8 & 8.1 & $56 \pm 27$ & $35 \pm 26$ & $2 \pm 2$ & $1 \pm 1$ & $2 \pm 2$ & $3 \pm 3$ \\
\hline
\end{tabular}


Table 5 Model 2: percentage protein (\% protein) contribution of humans at Mesolithic sites, modelled with FRUITS, assuming protein routing, incorporating six food sources

\begin{tabular}{|c|c|c|c|c|c|c|c|c|c|c|c|c|}
\hline ID & $\begin{array}{l}\text { Laboratory } \\
\text { ID }\end{array}$ & Site name & $\begin{array}{l}\text { Context/ } \\
\text { laboratory } \\
\text { ID }\end{array}$ & $\begin{array}{l}\text { Skeletal } \\
\text { element }\end{array}$ & $\begin{array}{l}\delta^{13} \mathrm{C} \\
\% o\end{array}$ & $\begin{array}{l}\delta^{15} \mathrm{~N} \\
\% o\end{array}$ & $\begin{array}{l}\text { Wild } \\
\text { plants }\end{array}$ & $\begin{array}{l}\text { Terrestrial } \\
\text { mammals }\end{array}$ & Shellfish & $\begin{array}{l}\text { Marine } \\
\text { fish }\end{array}$ & Seal & Freshwater \\
\hline $\mathrm{AH} 1$ & GrA-22431 & Aveline's Hole & M1.13/161 & Ulna & -19.4 & 9.0 & $75 \pm 13$ & $15 \pm 13$ & $2 \pm 2$ & $1 \pm 1$ & $2 \pm 2$ & $3 \pm 3$ \\
\hline $\mathrm{AH} 2$ & GrA-22938 & Aveline's Hole & M1.13/329 & Ulna & -19.4 & 8.7 & $55 \pm 25$ & $35 \pm 24$ & $2 \pm 2$ & $2 \pm 1$ & $2 \pm 2$ & $4 \pm 4$ \\
\hline $\mathrm{AH} 3$ & GrA-22555 & Aveline's Hole & M1.13/159 & Ulna & -19.3 & 8.4 & $77 \pm 17$ & $14 \pm 17$ & $2 \pm 2$ & $1 \pm 1$ & $2 \pm 2$ & $3 \pm 3$ \\
\hline AH4 & GrA-22546 & Aveline's Hole & M1.13/166 & Ulna & -21.0 & 8.5 & $82 \pm 11$ & $11 \pm 11$ & $1 \pm 1$ & $1 \pm 1$ & $2 \pm 2$ & $3 \pm 3$ \\
\hline AH5 & GrA-22428 & Aveline's Hole & M1.13/154 & Ulna & -19.6 & 9.3 & $72 \pm 10$ & $19 \pm 10$ & $2 \pm 2$ & $1 \pm 1$ & $2 \pm 2$ & $4 \pm 3$ \\
\hline AH6 & GrA-22433 & Aveline's Hole & M1.13/164 & Ulna & -19.1 & 8.2 & $79 \pm 15$ & $13 \pm 15$ & $2 \pm 2$ & $1 \pm 1$ & $2 \pm 2$ & $3 \pm 3$ \\
\hline $\mathrm{AH} 7$ & GrA-22422 & Aveline's Hole & M1.13/152 & Ulna & -19.3 & 9.0 & $66 \pm 24$ & $25 \pm 23$ & $2 \pm 2$ & $1 \pm 1$ & $2 \pm 2$ & $2 \pm 2$ \\
\hline AH8 & GrA-22429 & Aveline's Hole & M1.13/160 & Ulna & -19.5 & 8.8 & $70 \pm 22$ & $21 \pm 21$ & $2 \pm 2$ & $1 \pm 1$ & $2 \pm 2$ & $3 \pm 3$ \\
\hline AH9 & GrA-22557 & Aveline's Hole & M1.13/172 & Ulna & -19.8 & 8.1 & $82 \pm 13$ & $10 \pm 13$ & $2 \pm 2$ & $1 \pm 1$ & $2 \pm 2$ & $3 \pm 3$ \\
\hline AH10 & GrA-22621 & Aveline's Hole & M1.13/302 & Ulna & -19.0 & 10.3 & $47 \pm 25$ & $40 \pm 24$ & $3 \pm 2$ & $2 \pm 2$ & $3 \pm 2$ & $5 \pm 4$ \\
\hline AH11 & GrA-22432 & Aveline's Hole & M1.13/163 & Ulna & -19.9 & 8.4 & $78 \pm 17$ & $14 \pm 17$ & $2 \pm 2$ & $1 \pm 1$ & $2 \pm 2$ & $3 \pm 3$ \\
\hline AH12 & GrA-22548 & Aveline's Hole & M1.13/301 & Ulna & -20.0 & 7.7 & $86 \pm 8$ & $8 \pm 8$ & $2 \pm 1$ & $1 \pm 1$ & $2 \pm 2$ & $3 \pm 2$ \\
\hline AH13 & GrA-22552 & Aveline's Hole & M1.11/118 & Ulna & -19.3 & 9.2 & $66 \pm 24$ & $24 \pm 23$ & $2 \pm 2$ & $1 \pm 1$ & $2 \pm 2$ & $4 \pm 3$ \\
\hline AH14 & GrA-22558 & Aveline's Hole & M1.14/99 & Ulna & -20.0 & 9.0 & $71 \pm 19$ & $21 \pm 19$ & $2 \pm 1$ & $1 \pm 1$ & $2 \pm 2$ & $4 \pm 3$ \\
\hline AH15 & OxA-34338 & Aveline's Hole & M1.15.3 & Femur, R & -19.9 & 8.3 & $78 \pm 17$ & $14 \pm 17$ & $2 \pm 1$ & $1 \pm 1$ & $2 \pm 2$ & $3 \pm 3$ \\
\hline AH16 & OxA-34339 & Aveline's Hole & M1.14.55 & Tibia & -19.3 & 9.2 & $66 \pm 24$ & $24 \pm 23$ & $2 \pm 2$ & $1 \pm 1$ & $2 \pm 2$ & $4 \pm 3$ \\
\hline AH17 & OxA-35053 & Aveline's Hole & M1.11.111 & $\begin{array}{l}\text { Temporal, } \\
\text { R }\end{array}$ & -19.6 & 8.2 & $79 \pm 16$ & $13 \pm 16$ & $2 \pm 2$ & $1 \pm 1$ & $2 \pm 2$ & $3 \pm 3$ \\
\hline AH18 & OxA-35930 & Aveline's Hole & M1.11.220 & $\begin{array}{l}\text { Temporal, } \\
\text { R }\end{array}$ & -19.4 & 9.2 & $67 \pm 22$ & $24 \pm 22$ & $2 \pm 2$ & $1 \pm 1$ & $2 \pm 2$ & $4 \pm 3$ \\
\hline AH19 & OxA-35925 & Aveline's Hole & M1.11.141 & Petrous, L & -19.9 & 8.8 & $70 \pm 24$ & $22 \pm 24$ & $2 \pm 2$ & $1 \pm 1$ & $2 \pm 2$ & $3 \pm 3$ \\
\hline AH20 & OxA-34972 & Aveline's Hole & M1.11.141B & $\begin{array}{l}\text { Temporal, } \\
\text { R }\end{array}$ & -19.1 & 9.8 & $57 \pm 26$ & $32 \pm 26$ & $2 \pm 2$ & $2 \pm 2$ & $3 \pm 2$ & $4 \pm 4$ \\
\hline AH21 & OxA-34971 & Aveline's Hole & M1.11.2 & $\begin{array}{l}\text { Temporal, } \\
\text { R }\end{array}$ & -18.3 & 10.7 & $39 \pm 25$ & $45 \pm 25$ & $4 \pm 3$ & $2 \pm 2$ & $3 \pm 3$ & $6 \pm 5$ \\
\hline BH1 & OxA-1459 & Badger Hole & BH1 & Mandible & -20.3 & 8.5 & $79 \pm 16$ & $13 \pm 16$ & $1 \pm 1$ & $1 \pm 1$ & $2 \pm 2$ & $3 \pm 3$ \\
\hline $\mathrm{BH} 2$ & OxA-679 & Badger Hole & $\mathrm{BH} 2$ & Mandible & -20.5 & 9.6 & $51 \pm 26$ & $39 \pm 25$ & $2 \pm 2$ & $1 \pm 1$ & $2 \pm 2$ & $5 \pm 4$ \\
\hline BF1 & OxA-16865 & Bower Farm & Bower Farm 3 & Cranium & -20.8 & 2.8 & $96 \pm 2$ & $1 \pm 1$ & $1 \pm 1$ & $0 \pm 0$ & $1 \pm 1$ & $1 \pm 1$ \\
\hline CPQ1 & SUERC-84330 & $\begin{array}{l}\text { Cannington } \\
\text { Park Quarry }\end{array}$ & & Femur & -19.2 & 8.7 & $74 \pm 19$ & $17 \pm 19$ & $2 \pm 2$ & $1 \pm 1$ & $2 \pm 2$ & $3 \pm 3$ \\
\hline CPQ2 & SUERC-84331 & $\begin{array}{l}\text { Cannington } \\
\text { Park Quarry }\end{array}$ & & Femur & -19.7 & 8.6 & $75 \pm 17$ & $17 \pm 17$ & $2 \pm 2$ & $1 \pm 1$ & $2 \pm 2$ & $3 \pm 3$ \\
\hline $\mathrm{CC} 1$ & & Cnoc Coig & 8254 & $\begin{array}{r}\text { Cranial } \\
\text { frag? }\end{array}$ & -13.8 & 15.1 & $20 \pm 15$ & $22 \pm 13$ & $17 \pm 10$ & $11 \pm 8$ & $12 \pm 9$ & $18 \pm 13$ \\
\hline $\mathrm{CC} 2$ & & Cnoc & 8255 & Long bone? & -13.4 & 15.1 & $20 \pm 15$ & $20 \pm 12$ & $20 \pm 10$ & $11 \pm 8$ & $12 \pm 9$ & $17 \pm 13$ \\
\hline $\mathrm{CC} 3$ & OxA-29938 & Cnoc Coig & 8256 & Radius? & -13.3 & 15.0 & $19 \pm 15$ & $20 \pm 12$ & $22 \pm 11$ & $11 \pm 8$ & $12 \pm 9$ & $16 \pm 12$ \\
\hline $\mathrm{CC} 4$ & OxA-29939 & Cnoc Coig & 8257 & $\begin{array}{r}\text { Cranial } \\
\text { frag? }\end{array}$ & -14.1 & 15.4 & $18 \pm 14$ & $23 \pm 13$ & $15 \pm 9$ & $11 \pm 8$ & $13 \pm 9$ & $21 \pm 15$ \\
\hline $\mathrm{CC} 5$ & & Cnoc & 8258 & Cranial frag & -13.9 & 15.3 & $19 \pm 15$ & $22 \pm 13$ & $16 \pm 10$ & $12 \pm 8$ & $12 \pm 9$ & $19 \pm 14$ \\
\hline CC6 & & Cnoc Coig & 8260 & Vertebrae & -14.6 & 15.5 & $18 \pm 15$ & $24 \pm 13$ & $12 \pm 8$ & $11 \pm 8$ & $13 \pm 9$ & $23 \pm 15$ \\
\hline $\mathrm{CC} 7$ & & Cnoc Coig & 8266 & Vertebrae & -13.9 & 15.7 & $18 \pm 15$ & $21 \pm 13$ & $14 \pm 9$ & $13 \pm 9$ & $14 \pm 10$ & $21 \pm 14$ \\
\hline $\mathrm{CC} 8$ & & Cnoc Coig & 8267 & Unknown & -12.9 & 15.6 & $18 \pm 14$ & $17 \pm 11$ & $21 \pm 11$ & $14 \pm 9$ & $14 \pm 10$ & $16 \pm 12$ \\
\hline $\mathrm{CC} 9$ & & Cnoc Coig & $\begin{array}{c}\text { General Find } \\
1 \text { (GEN1) }\end{array}$ & Metacarpal? & -13.2 & 15.3 & $\mathrm{NM}$ & $\mathrm{NM}$ & $\mathrm{NM}$ & $\mathrm{NM}$ & $\mathrm{NM}$ & $\mathrm{NM}$ \\
\hline $\mathrm{CC} 10$ & GU-41836 & Cnoc Coig & & & -12.8 & 16.6 & $17 \pm 13$ & $15 \pm 10$ & $15 \pm 9$ & $18 \pm 11$ & $18 \pm 12$ & $17 \pm 12$ \\
\hline $\mathrm{CC} 11$ & GU-40827 & Cnoc Coig & & & -13.1 & 16.1 & $17 \pm 14$ & $17 \pm 11$ & $17 \pm 10$ & $15 \pm 10$ & $15 \pm 11$ & $18 \pm 13$ \\
\hline $\mathrm{CC} 12$ & & Cnoc Coig & 18,104 & Clavicle & -13.2 & 14.5 & $20 \pm 15$ & $20 \pm 12$ & $24 \pm 11$ & $9 \pm 7$ & $11 \pm 8$ & $15 \pm 11$ \\
\hline $\mathrm{CC} 13$ & OxA-8019 & Cnoc Coig & 17,157 & Clavicle & -12.3 & 16.0 & $17 \pm 13$ & $14 \pm 10$ & $23 \pm 11$ & $16 \pm 10$ & $16 \pm 11$ & $15 \pm 11$ \\
\hline $\mathrm{CC} 14$ & OxA-8014 & Cnoc Coig & 17,203 & Metacarpal & -12.0 & 14.7 & $18 \pm 14$ & $14 \pm 10$ & $33 \pm 12$ & $11 \pm 8$ & $12 \pm 9$ & $12 \pm 10$ \\
\hline $\mathrm{CC} 15$ & OxA-8004 & Cnoc Coig & 18,284 & Metacarpal & -12.0 & 17.0 & NM & NM & NM & NM & $\mathrm{NM}$ & NM \\
\hline $\mathrm{CC} 16$ & & Cnoc Coig & 18,089 & Frontal & -13.6 & 15.2 & $16 \pm 14$ & $22 \pm 13$ & $20 \pm 10$ & $12 \pm 8$ & $18 \pm 13$ & $20 \pm 16$ \\
\hline CNG1 & OxA-1281 & $\begin{array}{r}\text { Caisteal nan } \\
\text { Gillean II }\end{array}$ & & Metatarsal & -15.8 & 14.6 & $20 \pm 16$ & $33 \pm 16$ & $9 \pm 6$ & $7 \pm 6$ & $9 \pm 7$ & $22 \pm 15$ \\
\hline DR1 & OxA-7686 & Daylight Rock & $63.336 / 84.1$ & Mandible & -16.2 & 12.3 & $26 \pm 18$ & $43 \pm 15$ & $11 \pm 7$ & $4 \pm 3$ & $6 \pm 5$ & $10 \pm 9$ \\
\hline $\mathrm{FC} 1$ & OxA-8316 & Foxhole Cave & L2,FX97-41 & $\begin{array}{l}\text { Tooth, } \\
\text { canine }\end{array}$ & -20.0 & 11.3 & $33 \pm 24$ & $53 \pm 23$ & $2 \pm 2$ & $2 \pm 2$ & $3 \pm 3$ & $6 \pm 5$ \\
\hline $\mathrm{FC} 2$ & OxA-20838 & Foxhole Cave & FX08-8,C205 & $\begin{array}{l}\text { Lumbar } \\
\text { vertebra }\end{array}$ & -16.3 & 11.4 & $33 \pm 22$ & $42 \pm 20$ & $9 \pm 6$ & $3 \pm 3$ & $5 \pm 4$ & $7 \pm 6$ \\
\hline $\mathrm{FC} 3$ & OxA-26273 & Foxhole Cave & FX10-2,C407 & Vertebra & -16.8 & 11.7 & NM & $\mathrm{NM}$ & $\mathrm{NM}$ & NM & NM & NM \\
\hline $\mathrm{FC} 4$ & OxA-20835 & Foxhole Cave & FX08-5,C202 & $\begin{array}{l}\text { Lower M1, } \\
\quad \text { R }\end{array}$ & -15.4 & 12.2 & $27 \pm 20$ & $39 \pm 19$ & $14 \pm 8$ & $5 \pm 4$ & $6 \pm 5$ & $9 \pm 8$ \\
\hline FC5 & OxA-8315 & Foxhole Cave & L2,FX97-32 & Phalanx & -20.3 & 8.6 & $77 \pm 18$ & $15 \pm 19$ & $1 \pm 1$ & $1 \pm 1$ & $2 \pm 2$ & $4 \pm 3$ \\
\hline
\end{tabular}


Table 5 (continued)

\begin{tabular}{|c|c|c|c|c|c|c|c|c|c|c|c|c|}
\hline ID & $\begin{array}{l}\text { Laboratory } \\
\text { ID }\end{array}$ & Site name & $\begin{array}{l}\text { Context/ } \\
\text { laboratory } \\
\text { ID }\end{array}$ & $\begin{array}{l}\text { Skeletal } \\
\text { element }\end{array}$ & $\begin{array}{l}\delta^{13} \mathrm{C} \\
\% 0\end{array}$ & $\begin{array}{l}\delta^{15} \mathbf{N} \\
\% 0\end{array}$ & $\begin{array}{l}\text { Wild } \\
\text { plants }\end{array}$ & $\begin{array}{l}\text { Terrestrial } \\
\text { mammals }\end{array}$ & Shellfish & $\begin{array}{l}\text { Marine } \\
\text { fish }\end{array}$ & Seal & Freshwater \\
\hline FC6 & OxA-8318 & Foxhole Cave & L3,FX97-177 & Phalanx & -20.3 & 9.1 & $70 \pm 22$ & $22 \pm 22$ & $2 \pm 1$ & $1 \pm 1$ & $2 \pm 2$ & $4 \pm 3$ \\
\hline FC7 & OxA-8317 & Foxhole Cave & L1,FX97-59 & Tooth & -20.6 & 9.7 & $54 \pm 28$ & $37 \pm 28$ & $2 \pm 2$ & $1 \pm 1$ & $2 \pm 2$ & $4 \pm 4$ \\
\hline GL1 & Wk-30930 & Greylake & E22 & Cranium & -19.3 & 8.7 & $73 \pm 18$ & $17 \pm 19$ & $2 \pm 2$ & $1 \pm 1$ & $2 \pm 2$ & $3 \pm 3$ \\
\hline GL2 & Wk-30931 & Greylake & E23 & Cranium & -19.3 & 9.2 & $66 \pm 24$ & $24 \pm 23$ & $2 \pm 2$ & $1 \pm 1$ & $2 \pm 2$ & $4 \pm 3$ \\
\hline GL3 & OxA-25666 & Greylake & E22/23? & Mandible & -19.1 & 9.1 & NM & NM & NM & NM & NM & NM \\
\hline OY1 & OxA-7690 & Ogof-yr-Ychen & YY114 & Innominate & -15.2 & 15.6 & $18 \pm 14$ & $26 \pm 14$ & $9 \pm 7$ & $10 \pm 7$ & $12 \pm 9$ & $25 \pm 16$ \\
\hline OY2 & OxA-7691 & Ogof-yr-Ychen & YY115 & Innominate & -14.4 & 15.0 & $20 \pm 16$ & $25 \pm 14$ & $14 \pm 9$ & $10 \pm 7$ & $11 \pm 9$ & $19 \pm 14$ \\
\hline OY3 & OxA-22987 & Ogof-yr-Ychen & & Tibia & -14.5 & 15.2 & $20 \pm 15$ & $25 \pm 14$ & $13 \pm 8$ & $10 \pm 7$ & $12 \pm 9$ & $21 \pm 15$ \\
\hline OY4 & OxA-2574 & Ogof-yr-Ychen & B1 & Mandible & -14.9 & 15.4 & $19 \pm 15$ & $25 \pm 14$ & $11 \pm 7$ & $10 \pm 7$ & $12 \pm 8$ & $24 \pm 16$ \\
\hline OY5 & OxA-7742 & Ogof-yr-Ychen & B2 & Cranium & -15.7 & 15.6 & $17 \pm 14$ & $28 \pm 15$ & $7 \pm 6$ & $9 \pm 7$ & $12 \pm 9$ & $27 \pm 17$ \\
\hline OY6 & OxA-7741 & Ogof-yr-Ychen & $\mathrm{C}$ & Mandible & -16.9 & 12.9 & $25 \pm 19$ & $45 \pm 18$ & $7 \pm 5$ & $4 \pm 4$ & $6 \pm 5$ & $13 \pm 11$ \\
\hline OY7 & & Ogof-yr-Ychen & $\mathrm{C}^{*}$ & Cranium & -17.2 & 11.7 & $31 \pm 22$ & $46 \pm 20$ & $7 \pm 6$ & $4 \pm 3$ & $5 \pm 4$ & $8 \pm 7$ \\
\hline PAC1 & OxA-681 & $\begin{array}{l}\text { Paviland } \\
\text { (Goat's } \\
\text { Hole) Cave }\end{array}$ & $\begin{array}{l}\text { EM.603 } \\
\text { (Paviland } \\
\text { 2) }\end{array}$ & Humerus & -18.5 & 10.4 & $41 \pm 25$ & $46 \pm 25$ & $3 \pm 3$ & $2 \pm 2$ & $3 \pm 3$ & $5 \pm 4$ \\
\hline $\mathrm{PAC} 2$ & OxA-23801 & $\begin{array}{l}\text { Paviland } \\
\text { (Goat's } \\
\text { Hole) Cave }\end{array}$ & 0 & Humerus & -17.9 & 13.1 & $24 \pm 19$ & $48 \pm 19$ & $4 \pm 4$ & $4 \pm 3$ & $6 \pm 5$ & $14 \pm 11$ \\
\hline PAC3 & OxA-23802 & $\begin{array}{l}\text { Paviland } \\
\text { (Goat's } \\
\text { Hole) Cave }\end{array}$ & $\begin{array}{l}\text { EM.603 } \\
\text { (Paviland } \\
\text { 2) }\end{array}$ & Humerus & -18.2 & 11.6 & $32 \pm 24$ & $49 \pm 23$ & $4 \pm 4$ & $3 \pm 3$ & $4 \pm 4$ & $8 \pm 7$ \\
\hline POC1 & OxA-7687 & Potter's Cave & $\mathrm{PC} 1$ & Metacarpal & -17.5 & 11.9 & $31 \pm 22$ & $46 \pm 21$ & $6 \pm 5$ & $3 \pm 3$ & $5 \pm 4$ & $9 \pm 7$ \\
\hline $\mathrm{POC} 2$ & OxA-7688 & Potter's Cave & & Ulna & -17.3 & 13.1 & $24 \pm 18$ & $46 \pm 18$ & $6 \pm 5$ & $4 \pm 4$ & $7 \pm 5$ & $13 \pm 10$ \\
\hline SPS1 & OxA-8136 & $\begin{array}{l}\text { Staythorpe } \\
\text { Power } \\
\text { Station }\end{array}$ & Staythorpe 1 & Femur & -20.4 & 9.3 & $69 \pm 22$ & $23 \pm 22$ & $1 \pm 1$ & $1 \pm 1$ & $2 \pm 2$ & $4 \pm 3$ \\
\hline TH1 & & Thatcham III & & Humerus L & -21.9 & 8.4 & $85 \pm 10$ & $9 \pm 10$ & $1 \pm 1$ & $1 \pm 1$ & $1 \pm 1$ & $3 \pm 2$ \\
\hline TIL1 & OxA-18781 & Tilbury & $\begin{array}{c}\text { BMNH PA } \\
\text { SK 9, PV } \\
\text { M } 1913\end{array}$ & $\begin{array}{l}\text { Tibia shaft } \\
\text { R }\end{array}$ & -19.2 & 11.7 & $32 \pm 23$ & $52 \pm 22$ & $3 \pm 2$ & $2 \pm 2$ & $4 \pm 3$ & $8 \pm 7$ \\
\hline TOT1 & OxA-16457 & Totty Pot & TP1 & Femur & -19.7 & 10.3 & $50 \pm 25$ & $38 \pm 25$ & $2 \pm 2$ & $2 \pm 2$ & $2 \pm 2$ & $5 \pm 4$ \\
\hline MB1 & OxA-16604 & $\begin{array}{l}\text { Mewslade Bay } \\
\text { (Worm's } \\
\text { Head?) }\end{array}$ & $\begin{array}{l}\text { SM } \\
\quad 1919.41 .11\end{array}$ & Mandible & -19.0 & 9.1 & $73 \pm 17$ & $18 \pm 17$ & $2 \pm 2$ & $2 \pm 1$ & $2 \pm 2$ & $4 \pm 3$ \\
\hline MB2 & OxA-19845 & $\begin{array}{l}\text { Mewslade Bay } \\
\text { (Worm's } \\
\text { Head?) }\end{array}$ & $\begin{array}{l}\text { SM } \\
\quad 1919.41 .12\end{array}$ & Mandible & -19.0 & 9.8 & $60 \pm 26$ & $29 \pm 25$ & $3 \pm 2$ & $2 \pm 2$ & $2 \pm 2$ & $5 \pm 4$ \\
\hline WH1 & OxA-13131 & Worm's Head & $2001.4 \mathrm{H} / 4$ & Scapula & -19.3 & 10.2 & $53 \pm 25$ & $35 \pm 25$ & $2 \pm 2$ & $2 \pm 2$ & $3 \pm 2$ & $5 \pm 4$ \\
\hline WH2 & OxA-11128 & Worm's Head & WH1 & Ulna & -18.3 & 10.2 & $52 \pm 25$ & $34 \pm 25$ & $4 \pm 3$ & $2 \pm 2$ & $3 \pm 3$ & $5 \pm 4$ \\
\hline WH3 & OxA-16607 & Worm's Head & WH2 & Cranium & -19.0 & 11.4 & $34 \pm 23$ & $50 \pm 22$ & $3 \pm 3$ & $2 \pm 2$ & $3 \pm 3$ & $7 \pm 6$ \\
\hline WH4 & OxA-19844 & Worm's Head & 1924.6 .35 & Femur & -18.8 & 8.0 & $78 \pm 15$ & $13 \pm 15$ & $2 \pm 2$ & $1 \pm 1$ & $2 \pm 2$ & $3 \pm 3$ \\
\hline
\end{tabular}

$\mathrm{NM}-$ no model was generated

Park Quarry site, today located c. $1.9 \mathrm{~km}$ from the estuary of the River Parrett in southwest England (Fig. 1, site 13), may have been further from the coast during the Early Holocene.

\section{Late Upper Palaeolithic diets (models 1 and 3)}

Three LUP sites are included in our study. They comprise two inland sites, Gough's Cave and Sun Hole in southwest England, and one 'coastal' site, Kendrick's Cave in North Wales (Fig. 1, sites 3, 16 and 17). With RSL of c. -15 to $20 \mathrm{~m}$ along the North Wales coast between 12.0 and $11.7 \mathrm{ka}$ BP (Shennan et al. 2006, figure 7), Kendrick's Cave would have been further from the shoreline than it is today.
At Gough's Cave and Sun Hole, the vast majority of the diet was derived from terrestrial resources. Both protein routed (model 1) and nutrient scrambled (model 3 ) models indicate that at each of these sites the mean proportion of terrestrial (animal + plant) protein and food source calories was at least c. $89 \%$ (see SH1 in Tables 4 and 6), and possibly higher. Means and associated standard deviations suggest that the dietary contribution of marine and freshwater resources was likely negligible. Dependence on terrestrial resources is broadly consistent with previous interpretations of Lateglacial diet at Sun Hole and Gough's Cave (e.g. Richards et al. 2000; Stevens et al. 2010). 
Table 6 Model 3: percentage caloric (\%cal) intake of humans at Lateglacial sites, modelled with FRUITS, assuming nutrient scrambling, incorporating six food sources

\begin{tabular}{|c|c|c|c|c|c|c|c|c|c|c|c|}
\hline ID & $\begin{array}{l}\text { Laboratory } \\
\text { ID }\end{array}$ & Site name & Context/laboratory ID & $\begin{array}{l}\delta^{13} \mathrm{C} \\
\% o\end{array}$ & $\begin{array}{l}\delta^{15} \mathbf{N} \\
\% o\end{array}$ & $\begin{array}{l}\text { Wild } \\
\text { plants }\end{array}$ & $\begin{array}{l}\text { Terrestrial } \\
\text { herbivores }\end{array}$ & Shellfish & $\begin{array}{l}\text { Marine } \\
\text { fish }\end{array}$ & Seal & Freshwater \\
\hline GC1 & OxA-17848 & $\begin{array}{l}\text { Gough's } \\
\text { Cave }\end{array}$ & $\begin{array}{l}\text { (1.1/4) conjoin to frontal } \\
\text { (GC } 1987 \text { 169) }\end{array}$ & -19.3 & 7.6 & $42 \pm 32$ & $48 \pm 31$ & $3 \pm 3$ & $2 \pm 2$ & $2 \pm 2$ & $3 \pm 3$ \\
\hline $\mathrm{GC} 2$ & OxA-2236 & $\begin{array}{l}\text { Gough's } \\
\text { Cave }\end{array}$ & GC6; $1.1 / 3$ & -19.1 & 5.4 & $88 \pm 7$ & $7 \pm 7$ & $1 \pm 1$ & $1 \pm 1$ & $1 \pm 1$ & $1 \pm 1$ \\
\hline $\mathrm{GC} 3$ & OxA-17847 & $\begin{array}{l}\text { Gough's } \\
\text { Cave }\end{array}$ & GC M23.1/2 & -19.0 & 7.9 & $47 \pm 31$ & $43 \pm 30$ & $3 \pm 3$ & $2 \pm 1$ & $2 \pm 2$ & $3 \pm 3$ \\
\hline GC4 & OxA-17849 & $\begin{array}{l}\text { Gough's } \\
\text { Cave }\end{array}$ & GC 1987190 & -19.3 & 7.7 & $\mathrm{NM}$ & NM & $\mathrm{NM}$ & $\mathrm{NM}$ & $\mathrm{NM}$ & NM \\
\hline $\mathrm{KC} 1$ & OxA-7003 & $\begin{array}{l}\text { Kendrick's } \\
\text { Cave }\end{array}$ & 57 & -17.9 & 13.8 & $19 \pm 16$ & $35 \pm 16$ & $6 \pm 5$ & $6 \pm 5$ & $7 \pm 6$ & $27 \pm 15$ \\
\hline $\mathrm{KC} 2$ & OxA-7004 & $\begin{array}{l}\text { Kendrick's } \\
\text { Cave }\end{array}$ & 59 & -18.0 & 13.4 & $20 \pm 18$ & $39 \pm 16$ & $6 \pm 5$ & $6 \pm 5$ & $7 \pm 6$ & $23 \pm 14$ \\
\hline $\mathrm{KC} 3$ & OxA-6114 & $\begin{array}{l}\text { Kendrick's } \\
\text { Cave }\end{array}$ & 60 & -17.7 & 13.9 & $19 \pm 17$ & $34 \pm 16$ & $6 \pm 6$ & $6 \pm 5$ & $8 \pm 6$ & $26 \pm 15$ \\
\hline $\mathrm{KC4}$ & OxA-7002 & $\begin{array}{l}\text { Kendrick's } \\
\text { Cave }\end{array}$ & 69 & -18.1 & 13.7 & $19 \pm 16$ & $36 \pm 16$ & $6 \pm 5$ & $5 \pm 5$ & $7 \pm 6$ & $27 \pm 14$ \\
\hline $\mathrm{KC5}$ & OxA-17089 & $\begin{array}{l}\text { Kendrick's } \\
\text { Cave }\end{array}$ & 74 & -17.7 & 13.2 & $20 \pm 17$ & $40 \pm 16$ & $7 \pm 6$ & $6 \pm 5$ & $7 \pm 6$ & $20 \pm 13$ \\
\hline SH1 & OxA-19557 & Sun Hole & M5.13/24 & -18.8 & 8.1 & $42 \pm 30$ & $47 \pm 28$ & $3 \pm 3$ & $2 \pm 2$ & $2 \pm 2$ & $4 \pm 3$ \\
\hline
\end{tabular}

$\mathrm{NM}$ —no model was generated

Previous LMM studies of the Kendrick's Cave population have led to conflicting dietary interpretations. Richards et al. (2005:392) proposed that the carbon stable isotope values were consistent with 'a diet where approximately $30 \%$ of dietary protein was from marine resources' and also argued that the nitrogen stable isotope values indicated 'a mix between protein from terrestrial herbivores, such as Cervus elaphus, and marine mammals, such as seals'. Bocherens and Drucker (2006) highlighted some of the limitations of Richards et al.'s (2005) analysis: the failure to incorporate uncertainty in $\delta^{13} \mathrm{C}$ trophic level shifts, the small sample size of the terrestrial mammals used as a food source baseline and the absence of freshwater resources from the food sources modelled. Bocherens and Drucker (2006) re-evaluated the Kendrick's Cave data using a mixing model for four food sources - terrestrial herbivores, salmon, seal and freshwater fish. They concluded that more than one dietary model fits the data, asserting that 'it is not necessary to incorporate herbivore meat to explain the human isotopic values' (Bocherens and Drucker 2006:441) and diets based entirely on aquatic resources (both freshwater and marine) could account for the stable isotope signatures of the Kendrick's Cave humans.

Our FRUITS models of diet for Kendrick's Cave based on six food groups differ from previous mixing model-based interpretations. On model 3 (scrambled routing), terrestrial food sources made the greatest caloric contribution to diet (with a uniform plant \%cal contribution to the diet of each individual in the range $19 \pm 16 \%$ to $20 \pm 18 \%$ and terrestrial herbivore $\%$ cal contribution from $34 \pm 16 \%$ to $40 \pm 16 \%$ ). Freshwater resources constituted a secondary but significant dietary component (c. $20 \pm 13 \%$ to $27 \pm 15 \%$ ), though marine resources were also important (with broadly similar \%cal proportions in each individual's whole diet of c. $6 \pm 5 \%$ shellfish, $6 \pm 5 \%$ marine fish and $7 \pm 6 \%$ sea mammal). The corresponding estimates generated by model 1 (protein routed) are plants c. 26 $\pm 18 \%$ to $29 \pm 21 \%$, terrestrial herbivores from $30 \pm 16 \%$ to $37 \pm 18 \%$, freshwater sources from $23 \pm 13 \%$ to $31 \pm 15 \%$, shellfish c. $3 \pm 3 \%$, fish c. $4 \pm 3 \%$ and sea mammal $6 \pm 5 \%$.

Here, it is also worth noting that remains of fish and other aquatic foods are generally scarce on Lateglacial sites in Britain, perhaps due to taphonomic and recovery biases (Bocherens and Drucker 2006). Fish remains are generally more friable than mammal bones (Nichol and Wild 1984) and this, combined with the fact that many LUP and Mesolithic sites were excavated before fine recovery techniques were widely practised, limits the chances of recovery. Many coastal sites in the southern half of Britain older than c. $7.0 \mathrm{ka}$ BP were probably submerged during the post-glacial marine transgression, although the rare find of the scales of lemon sole (Microstomus kitt) from Pinhole Cave, Creswell Crags, northern England (Armstrong 1932) is confirmation that marine resources were being exploited at least occasionally during the LUP.

\section{Mesolithic diets (models 2 and 4)}

Human stable isotope data from 18 Mesolithic sites were analysed, seven dated to the Late Mesolithic and 11 to the 


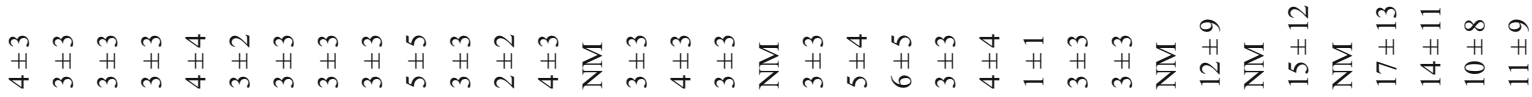

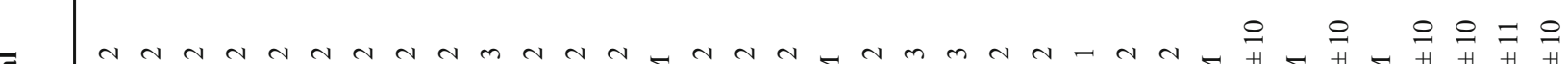

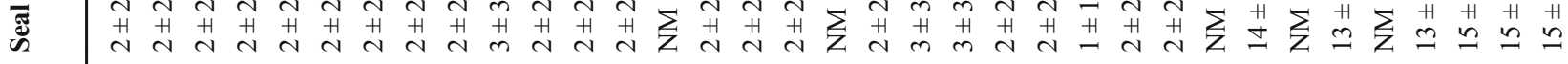
里

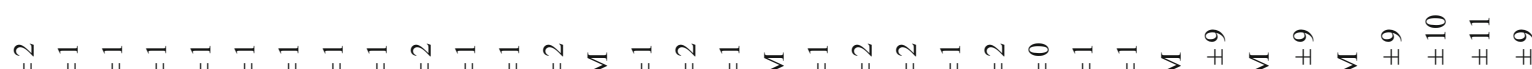

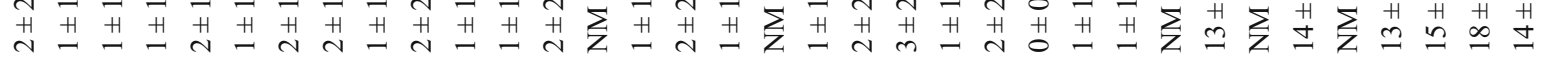

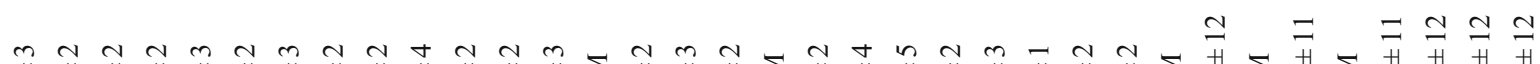

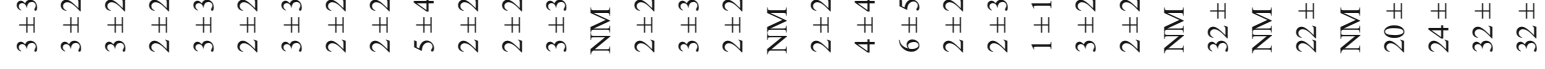

กี่ง

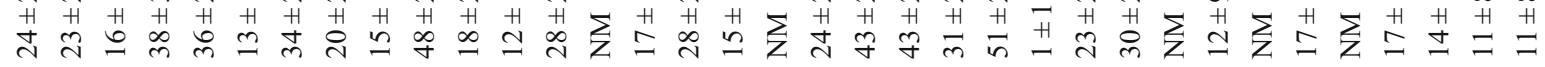
ป઼

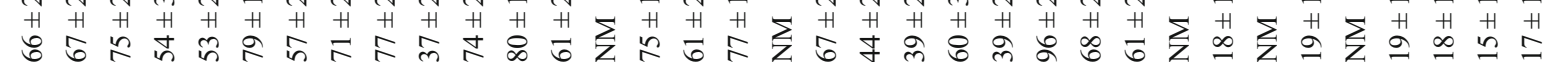
Z 앙

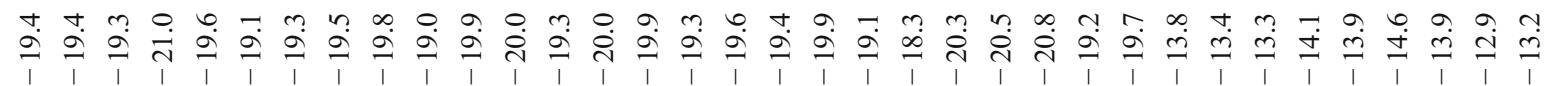

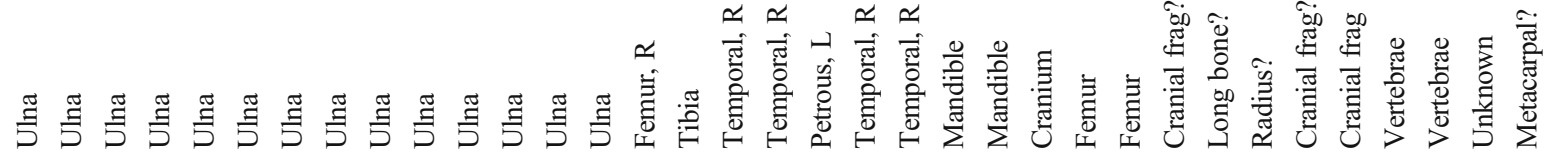

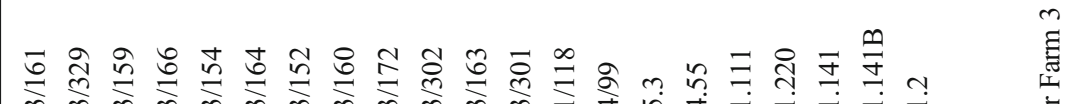

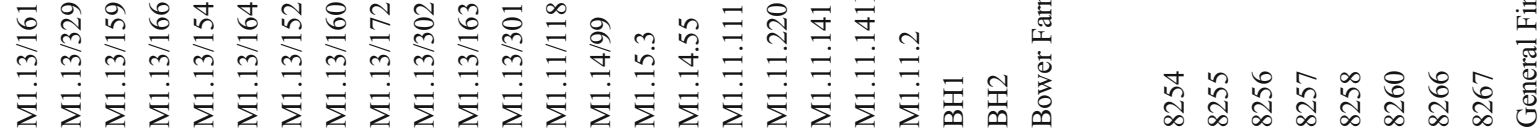

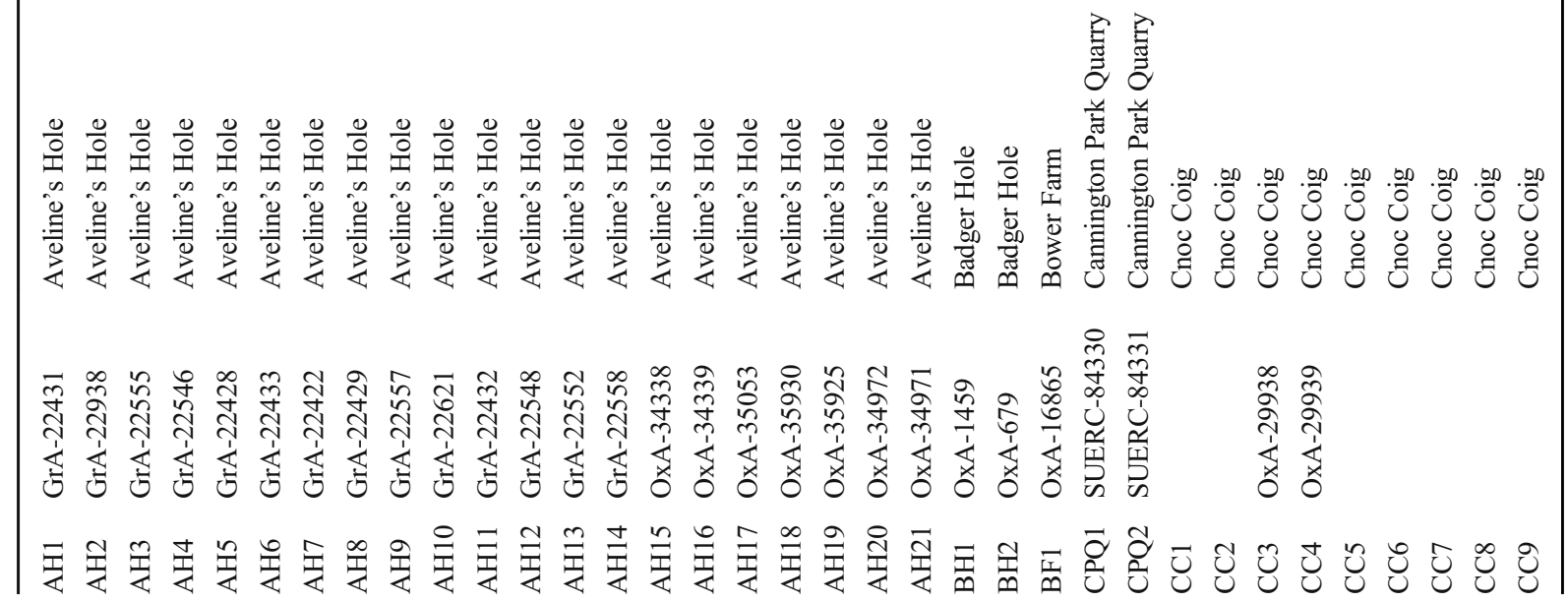


离|

垔

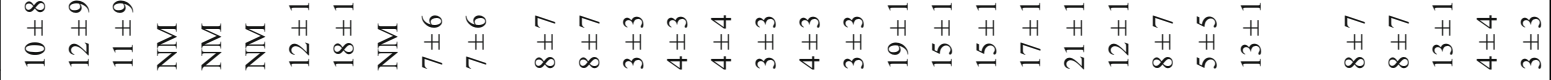

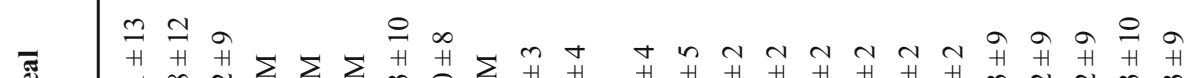

פ⿹

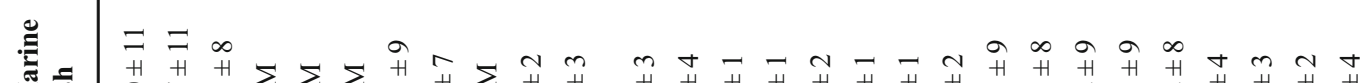

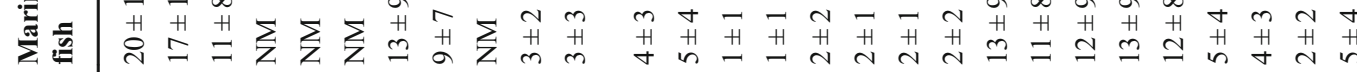

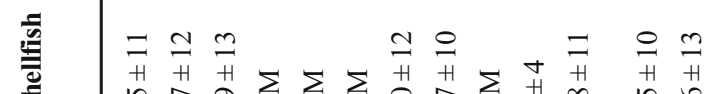

के

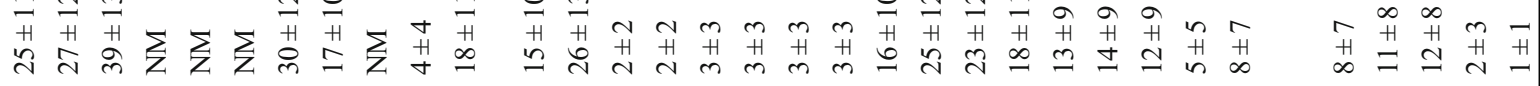

焉

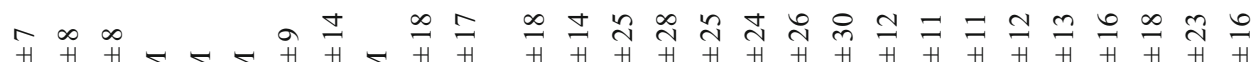

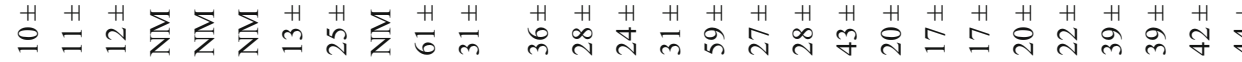

구을

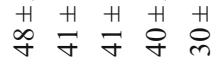

들

$\simeq$

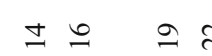

今

สำ

สิ

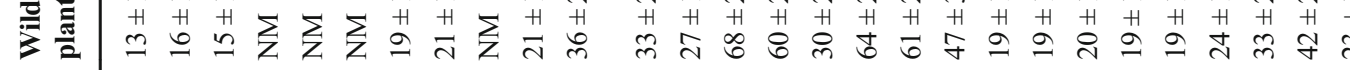

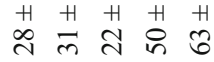

$\underbrace{2}_{\substack{2 \\ 0}}$

苞

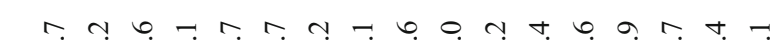

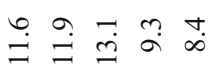

$\sum_{\infty}^{\infty}$

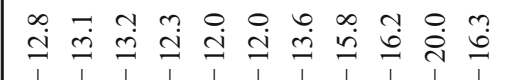

車

ก ก $m$ เ

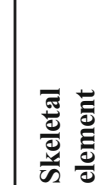

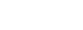

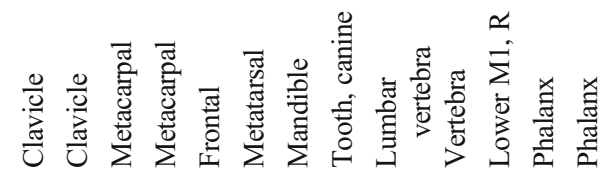

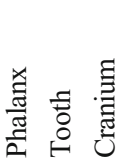

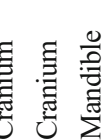

竎

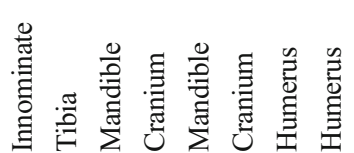

总总

$\cong$

हैं

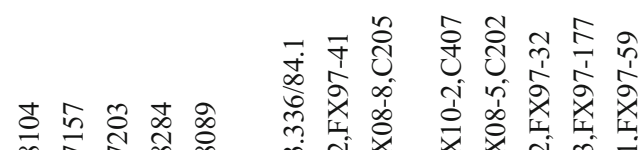

ஸें

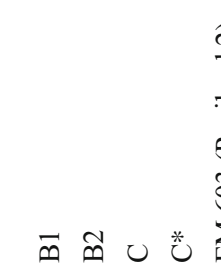

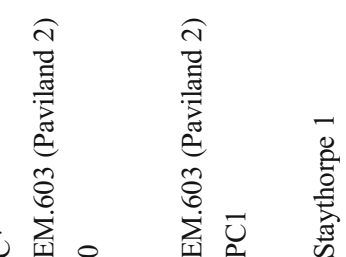

苞

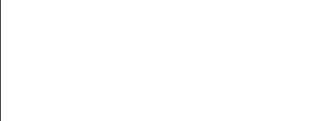

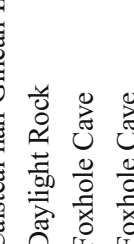

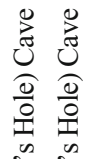

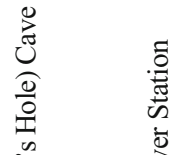

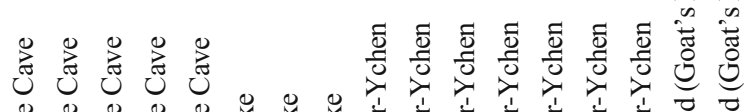

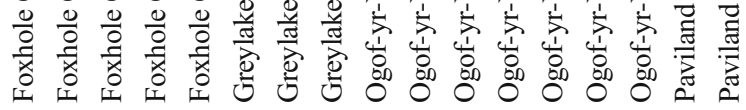

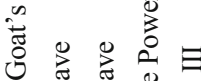

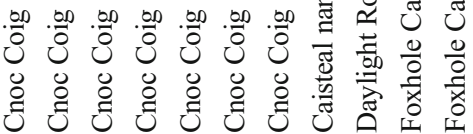

उ $\bigcup_{\infty}$ :

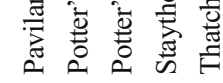

要

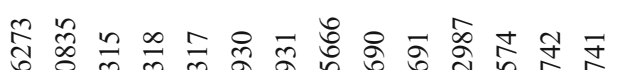

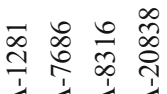

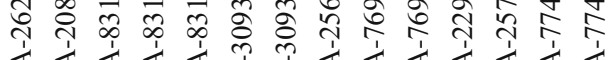

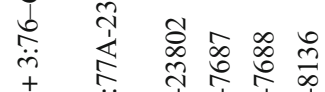

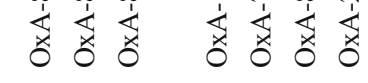

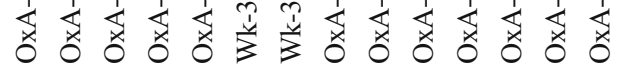

荐 $\begin{gathered}+ \\ 0\end{gathered}$

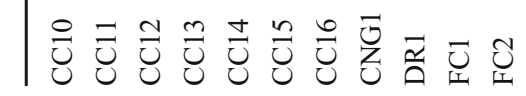

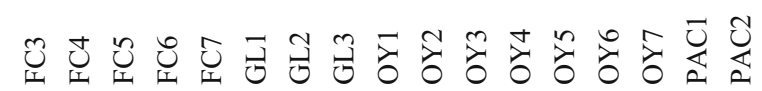

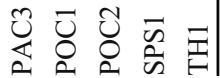


Early Mesolithic (n.b. the specimens attributed to a site at Mewslade Bay are assumed to have originated from Worm's Head; cf. Meiklejohn et al. 2011). FRUITS modelling suggests significant site diversity in dietary intake, ranging from groups whose diets were largely based on terrestrial food sources to those with mixed diets that included terrestrial, marine and freshwater foods.

Coastal hunter-gatherers at Cnoc Coig, Oronsay, had the highest proportion of diet derived from marine foods (with the highest individual mean intake of marine protein in sample CC14 of c. $56 \%$ (shellfish $33 \pm 12 \%$, fish $11 \pm 8 \%$ and seal $12 \pm 9 \%$ ) in model 2 and of whole diet calories in sample CC10 of c. $66 \%$ (shellfish $25 \pm 11 \%$, fish $20 \pm 11 \%$ and seal $21 \pm 13 \%$ ) in model 4). Previous interpretations of diet at Cnoc Coig have emphasized the importance of marine resources with linear mixing models based on the $\delta^{13} \mathrm{C}$ and $\delta^{15} \mathrm{~N}$ values used to suggest that up to $100 \%$ of dietary protein was derived from marine foods (cf. Richards and Mellars 1998; Schulting and Richards 2002b; Charlton et al. 2016). Our FRUITS models, using essentially the same data but modelling for food source calories, suggest that terrestrial foods made a significant contribution to overall diet (with individual intake of terrestrial protein up to c. $42 \%$ in model 2 (e.g. sample CC1with $20 \pm 15 \%$ plant and $22 \pm 13 \%$ herbivore/omnivore protein) and up to c. $36 \%$ of total calories in model 4 (e.g. sample CC4 with $19 \pm 15 \%$ plant and $17 \pm 11 \%$ herbivore/omnivore calories)). In most samples, the proportion of plant versus terrestrial mammal food in the diet is roughly equal. Both FRUITS models highlight the dietary importance of shellfish as well as fish and sea mammals. The nutrient scrambled model suggests some individuals obtained more than one-third of their calories from shellfish.

The results from Cnoc Coig hint at individual variation in dietary intake (see Fig. 2). For example, the nutrient scrambled model suggests that individual CC12 may have obtained up to $39 \pm 13 \%$ of calories from shellfish, while individual CC6 consumed a much smaller proportion of shellfish, c. 20 $\pm 11 \%$. However, individual variation in dietary intake at Cnoc Coig may be inflated by uncertainty over the age-atdeath of the individuals sampled. Although this may also be an issue at other sites, the use of ZooMS to identify small, undiagnostic fragments of bone suggests this may be especially problematic at Cnoc Coig. A nursing effect in infants and young children causes ${ }^{13} \mathrm{C}$ - and ${ }^{15} \mathrm{~N}$-enrichment above maternal values of up to c. $1 \%$ and c. $3 \%$, respectively (Fuller et al. 2006). Additionally, increased protein requirements during adolescence may result in reduced fractionation of dietary ${ }^{14} \mathrm{~N} /{ }^{15} \mathrm{~N}$ (e.g. Waters-Rist and Katzenberg 2010). Bone collagen laid down in adolescence may, therefore, have lower $\delta^{15} \mathrm{~N}$ than at other stages of life in individuals with monotonous 'lifetime' diets. 
Fig. 1 Sites with Late Upper Palaeolithic or Mesolithic human remains included in this study: 1 . Cnoc Coig; 2. Caisteal nan Gillean II; 3. Kendrick's Cave; 4. Pontnewydd Cave; 5. Bower Farm; 6. Staythorpe; 7. Potter's Cave; 8. Ogof-yr-Ychen; 9. Daylight Rock; 10. Worm's Head (Mewslade Bay); 11. Foxhole Cave; 12. Goat's Hole; 13. Cannington Park Quarry; 14. Greylake, 15. Aveline's Hole; 16. Gough's Cave; 17. Sun Hole; 18. Totty Pot; 19. Badger Hole; 20. Thatcham III; 21. Tilbury

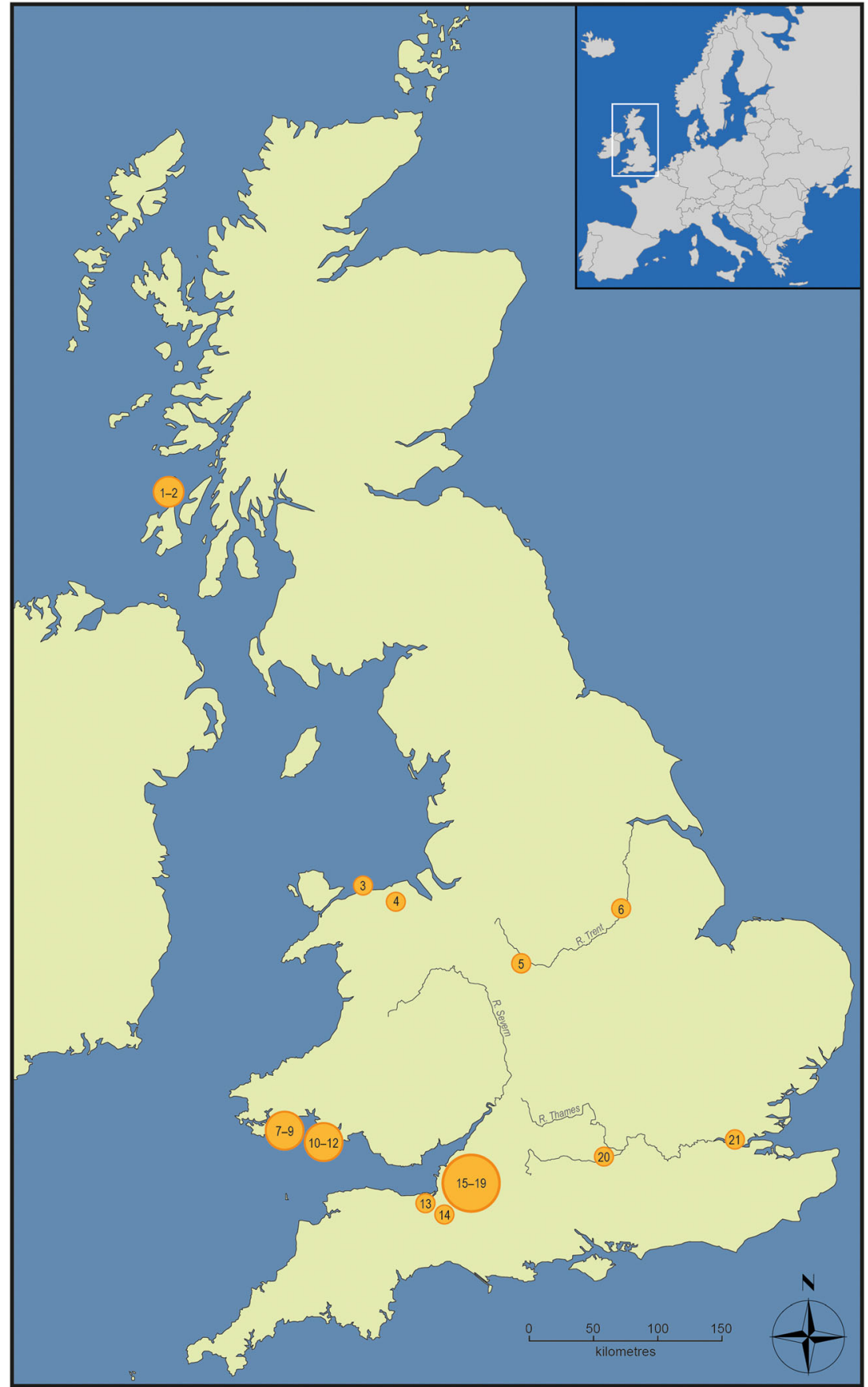

Similar intra-site variability is evident at Ogof-yr-Ychen in South Wales. However, in this case, terrestrial foods constitute dietary staples; one individual analysed (OY7) had whole diet calorie proportions that were $33 \pm 22 \%$ plant, $39 \pm 18 \%$ terrestrial mammal, $12 \pm 9 \%$ shellfish, $4 \pm 3 \%$ marine fish, $5 \pm 4 \%$ seal and $8 \pm 7 \%$ freshwater (model 4 ).

Schulting and Richards (2002a, Table 1) proposed that the main dietary protein source was marine $63-72 \%$ at Ogof-yrYchen and that individuals with particularly high $\delta^{15} \mathrm{~N}$ had consumed significant quantities of seal meat. Our FRUITS models suggest a mixed diet with a particular emphasis on freshwater fish. For each individual, the mean estimate of total calories obtained from shellfish $(12 \pm 9 \%$ to $25 \pm 12 \%$ in model 4$)$ is equal to or exceeds that from seals ( $5 \pm 4 \%$ to $13 \pm 10 \%$ in model 4). Schulting and Richards (2002a:1017) observed that certain individuals from Ogof-yr-Ychen 'show a more balanced use of marine and terrestrial resources that could imply seasonal movements; inland groups may have maintained social links with coastal communities allowing them access at certain times of the year'. Our FRUITS estimates 


\section{Whole diet}

a

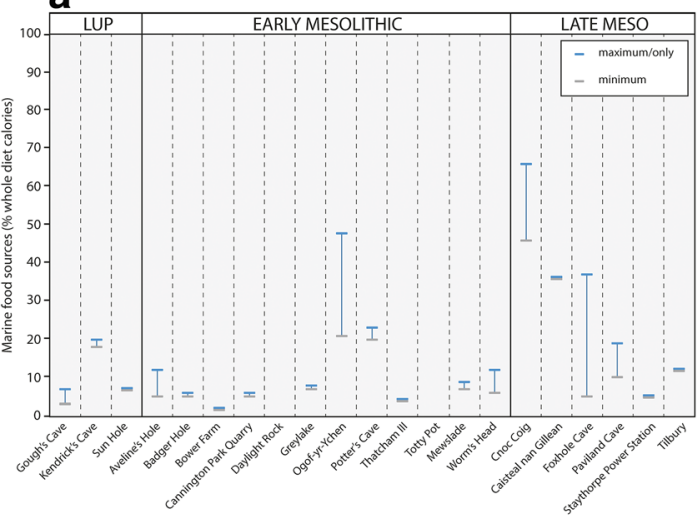

b
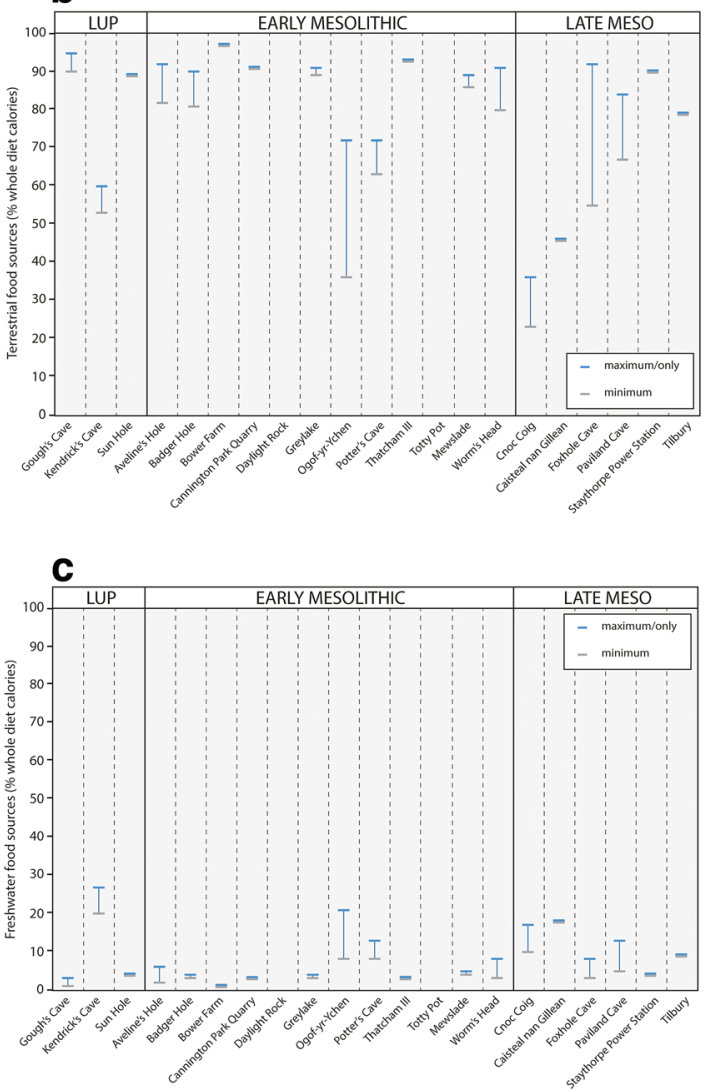

Fig. 2 Summary of FRUITS models for each site, showing the maximum and minimum, or in the case of sites with a single sample the sole, mean estimated food source contribution to diet for (a) \% marine whole diet calorie contribution, (b) \% terrestrial whole diet calorie contribution, (c)

suggest that all but two of the samples analysed from Ogof-yrYchen had broadly similar mixed diets. The two samples, $\mathrm{C}$ and $\mathrm{C}^{*}$, which may come from one individual, have a slightly higher proportion of terrestrial food calories in diet than the other samples, though both show similar proportions of marine food calorie intake to the other samples from Ogof-yrYchen - the main difference is in the contribution to diet of freshwater fish.

\section{Protein}
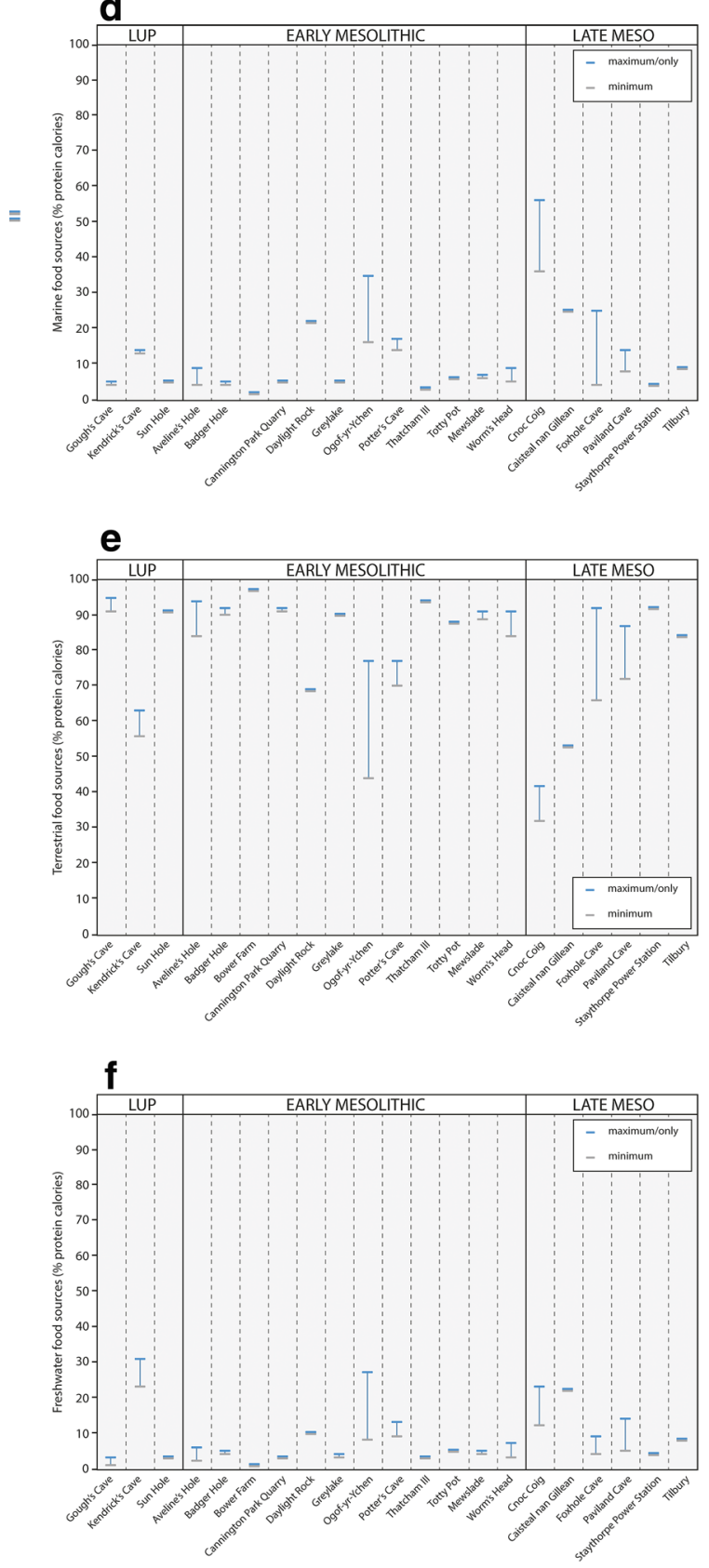

$\%$ freshwater whole diet calorie contribution, (d) \% marine protein contribution, (e) $\%$ terrestrial protein contribution and (f) $\%$ freshwater protein contribution

At all of the other Mesolithic sites analysed, terrestrial food sources dominated the diet. However, there is variation between and within groups in the proportions of plant and herbivore/omnivore food sources consumed. At Aveline's Hole, for example, the contribution of plant foods to protein was $39 \pm 25 \%$ to $86 \pm 8 \%$ (model 2 ) and to whole diet $39 \pm$ $25 \%$ to $80 \pm 17 \%$ (model 4 ), while that of terrestrial mammals to protein was $8 \pm 8 \%$ to $45 \pm 25 \%$ (model 2 ) and to whole diet 
$12 \pm 16 \%$ to $48 \pm 25 \%$ (model 4 ). The individual from Bower Farm had a diet with a very high proportion $(96 \pm 2 \%)$ of plant foods unless the unusually low $\delta^{15} \mathrm{~N}$ value of $2.8 \%$ is a reflection of long-term nutritional stress or disease.

\section{Wider issues}

We encountered a number of technical and practical issues relating to Bayesian modelling of the British data:

- The FRUITS software (v. 3.0 beta) would fail to generate model outputs for certain site datasets or even individual samples, most particularly where we attempted to introduce prior information into our models. Partly for this reason, our dietary models do not include the prior assumption of 5-40\% of protein intake recommended by Fernandes et al. (2014). Initially, we attributed these problems to the fact that the number of food sources (six) far exceeded the number of proxies (two $-\delta^{13} \mathrm{C}, \delta^{15} \mathrm{~N}$ ). To investigate this issue further, FRUITS models were generated for four food sources: plants, terrestrial herbivores/ omnivores, freshwater fish and grouped marine resources. The results were broadly similar in terms of food proportions in diet, although overall there was an increase in the proportion of plants in diet compared to the six-foodsource models and a corresponding decrease in the proportion of marine food. Roughly the same proportion of samples failed to generate model outputs in both the fourand six-food-source models. It is therefore tentatively suggested here that the problems encountered with model generation and also possibly the large one-sigma standard deviations of certain modelled diets may result from the inadequacies in food source data rather than the number of proxies or the number of food sources.

- A third proxy, sulphur stable isotope ratios $\left(\delta^{34} \mathrm{~S}\right)$, has been incorporated into dietary models. Arguably, $\delta^{34} \mathrm{~S}$ is a particularly useful aid to dietary reconstruction in situations where freshwater food sources were available (e.g. Nehlich et al. 2010; although see Bonsall et al. 2015 for caveats). However, measurements of $\delta^{34} \mathrm{~S}$ have not been undertaken routinely for Lateglacial or Mesolithic human remains. Moreover, the utility of $\delta^{34} \mathrm{~S}$ as a dietary discriminant for the sites included in this study is uncertain. Seaspray can deposit oceanic sulphate considerable distances inland (Thode 1991). This results in soil and in turn terrestrial food web, $\delta^{34} \mathrm{~S}$ values mirroring oceanic $\delta^{34} \mathrm{~S}$. The extent and impacts of sea-spray on food web $\delta^{34} \mathrm{~S}$ in the Lateglacial and Early Holocene would be contingent on palaeogeography.

- Some of our FRUITS model predictions of plant food intake in British LUP and Mesolithic populations (Tables 4, 5, 6 and 7) are high in comparison to estimates of $26-45 \%$ dependence on plant foods among recent temperate zone hunter-gatherers (Cordain et al. 2000; Table 2) derived from an extensive review of ethnographic evidence, notwithstanding that Cordain's data are based on food weight rather than caloric content. The FRUITS estimates of dependence on plant foods of $42 \pm 30 \%$ at Sun Hole and up to $88 \pm 7 \%$ at Gough's Cave among the LUP populations (in model 3-see Table 6) are particularly surprising given that during the early part of the Bølling-Allerød Interstadial, trees that produce high nutritional value nuts (e.g. acorn, hazelnut) were absent from the British flora (cf. Ince 1996; Hill et al. 2008).

- These observations highlight the current lack of wild plant food protein and energy $\delta^{13} \mathrm{C}$ data from Britain. Our FRUITS models were constructed using data from domesticated plant food remains in Bogaard et al. (2013), which may not be appropriate. Further research is vital to determine stable isotope values for local edible wild plants including hazelnuts, which may have been subject to a canopy effect (van der Merwe and Medina 1991).

- A related point is that FRUITS assumes the contribution to dietary carbohydrate $(\mathrm{CH})$ from animal tissues is negligible (Fernandes et al. 2014) and (by extension) is obtained almost exclusively from plants. However, $\mathrm{CH}$ occurs in some organs (e.g. liver) of terrestrial and marine mammals and some species of shellfish, especially bivalves such as cockles, oysters and mussels (USDA database). Honeytechnically, an animal (insect) product - is also a rich $\mathrm{CH}$ source. Moreover, post-glacial hunter-gatherers probably consumed nearly all the edible parts of the animals they exploited for food (meat, organs, bone marrow, etc.), providing higher amounts of $\mathrm{CH}$ and fat than meat alone. It follows that our FRUITS models may overestimate the proportion of diet obtained from plants.

- Our FRUITS estimates for the contribution of freshwater resources to post-glacial hunter-gatherer diets are also unexpected. Populations living near one of the longest rivers in Britain - at Bower Farm and Staythorpe in the valley of the River Trent-show relatively minor contributions from freshwater fish $(1 \pm 1 \%$ and $4 \pm 3 \%$, respectively, in both models 2 and 4) (Tables 5 and 7). Likewise, freshwater resources were apparently unimportant at the Early Holocene wetland site of Thatcham III in southern England - although the single human bone from this site was found in a secondary context (Churchill 1963) and so the Mesolithic dating is not secure. It is possible, however, that our model estimates are inaccurate; the lack of local baseline data for freshwater fish necessitated using stable isotope data for freshwater fish from a very broad geographic area (for a review of variation in freshwater fish stable isotope values, see Guiry 2019). By contrast, individuals from Ogof-yr-Ychen were modelled to have had a relatively high proportion of freshwater food sources in their diets despite the lack of large bodies of fresh water 
on or near Caldey Island. This may relate to group movement (see Preston and Kador 2018 for a review of mobility models among British hunter-gatherers) and exploitation of resources across the broader Carmarthen Bay area and beyond. More particularly, it may reflect a further complexity in modelling hunter-gatherer diets - the distinctive isotopic signatures of estuarine resources. The Carmarthen Bay area encompasses extensive estuarine habitats. Estuaries typically support a greater abundance and diversity of potential food resources than other coastal habitats, including fish, shellfish, birds and edible plants, which made them particularly attractive to post-glacial huntergatherers (Bonsall 1981). Fish and shellfish harvested from estuaries may have $\delta^{13} \mathrm{C}$ intermediate between marine and freshwater values (cf. Thornton and McManus 1994), and diets composed largely of estuarine resources may mimic mixed diets of marine and freshwater food sources. This dietary scenario may also hold for the sample from Caisteal nan Gillean II, Oronsay, which had similar $\delta^{13} \mathrm{C}$ and $\delta^{15} \mathrm{~N}$ values to some of the individuals from Ogof-yr-Ychen.

- Research is also required to establish tissue offsets in marine mammals. In terrestrial mammals, muscle tissue is generally ${ }^{15} \mathrm{~N}$-enriched relative to bone collagen; however, this was not evident in Hobson et al.'s (1996) study of seal tissues, though their data are limited in number.

\section{Conclusions}

FRUITS modelling of the diets of post-glacial hunter-gatherers in Great Britain, based on bulk bone collagen stable Cand $\mathrm{N}$-isotope values, suggests there was significant spatial and temporal variability in subsistence practices.

At the three LUP sites included in our analysis (Gough's Cave and Sun Hole in south-west England, and Kendrick's Cave in North Wales), terrestrial food sources appear to have been the dietary staples, although at Kendrick's Cave freshwater resources also made a significant contribution. While marine foods may have contributed to diet at all three sites, they constituted at most a very minor resource.

Previous stable isotope studies of Mesolithic diets have tended to emphasise the role of aquatic (primarily marine) foods and perhaps underestimated the contribution of plant foods. Models of food source calorie contribution to diet indicate that only two coastal sites in this study (Cnoc Coig and Caisteal nan Gillian II on the island of Oronsay, off the west coast of Scotland) show a clear emphasis on aquatic resources, although terrestrial resources also contributed significantly to caloric intake. In contrast, FRUITS models of the Mesolithic populations living along the South Wales coast suggest diets where often the majority of calories were drawn from terrestrial resources, although in at least one case (Ogof-yrYchen) aquatic resources appear to have been important. However, these models are treated cautiously in light of the confounding isotope values of estuarine resources.

Predictably, Mesolithic inland sites show a heavy emphasis on terrestrial (animal and plant) resources, though one surprising outcome of our model predictions is the limited evidence for the use of freshwater resources at sites in the vicinity of large rivers or wetlands (Bower Farm, Staythorpe and Thatcham III).

We are acutely aware of the shortcomings of the research presented here. We were unable to generate FRUITS models for certain sites and individuals, possibly related to the small number of proxies relative to food sources and/or deficiencies in the food source data. Moreover, our model predictions are likely biased by inadequate baseline $\delta^{13} \mathrm{C}$ and $\delta^{15} \mathrm{~N}$ data for wild terrestrial plant, freshwater and estuarine resources, in particular. Accurately quantifying the proportion of aquatic resources in diet is also crucial in calculating radiocarbon reservoir offsets.

Open Access This article is licensed under a Creative Commons Attribution 4.0 International License, which permits use, sharing, adaptation, distribution and reproduction in any medium or format, as long as you give appropriate credit to the original author(s) and the source, provide a link to the Creative Commons licence, and indicate if changes were made. The images or other third party material in this article are included in the article's Creative Commons licence, unless indicated otherwise in a credit line to the material. If material is not included in the article's Creative Commons licence and your intended use is not permitted by statutory regulation or exceeds the permitted use, you will need to obtain permission directly from the copyright holder. To view a copy of this licence, visit http://creativecommons.org/licenses/by/4.0/.

\section{References}

Amkreutz L, Verpoorte A, Waters-Rist A, Niekus M, van Heekeren V, van der Merwe A, van der Plicht H, Glimmerveen J, Stapert D, Johansen L (2018) What lies beneath... Late Glacial human occupation of the submerged North Sea landscape. Antiquity 92:22-37. https://doi.org/10.15184/aqy.2017.195

Armstrong AL, (1932) Excavations in the Pin Hole Cave, Creswell Crags, Derbyshire. Proceedings of the Prehistoric Society of East Anglia 6(4):330-334

Barrett JH, Orton D, Johnstone C, Harland J, Van Neer W, Ervynck A, Roberts C, Locker A, Amundsen C, Enghoff IB, Hamilton-Dyer S, Heinrich D, Hufthammer AK, Jones AKG, Jonsson L, Makowiecki D, Pope P, O'Connell TC, de Roo T, Richards M (2011) Interpreting the expansion of sea fishing in medieval Europe using stable isotope analysis of archaeological cod bones. J Archaeol Sci 38:1516-1524. https://doi.org/10.1016/j.jas.2011.02.017

Berg OK, Bremset G (1998) Seasonal changes in the body composition of young riverine Atlantic salmon and brown trout. J Fish Biol 52: 1272-1288. https://doi.org/10.1111/j.1095-8649.1998.tb00971.x 
Bocherens H, Drucker D (2006) Isotope evidence for paleodiet of late upper Paleolithic humans in Great Britain: a response to Richards et al. (2005). J Hum Evol 51:440-442. https://doi.org/10.1016/j. jhevol.2005.12.014

Bocherens H, Polet C, Toussaint M (2007) Palaeodiet of Mesolithic and Neolithic populations of Meuse Basin (Belgium): evidence from stable isotopes. J Archaeol Sci 34:10-27. https://doi.org/10.1016/j. jas.2006.03.011

Bocherens H, Grandal-d'Anglade A, Hobson KA (2014) Pitfalls in comparing modern hair and fossil bone collagen $\mathrm{C}$ and $\mathrm{N}$ isotopic data to reconstruct ancient diets: a case study with cave bears (Ursus spelaeus). Isot Environ Health Stud 50:291-299. https://doi.org/ 10.1080/10256016.2014.890193

Bogaard A, Fraser R, Heaton THE, Wallace M, Vaiglova P, Charles M, Jones G, Evershed RP, Styring AK, Andersen NH, Arbogast R-M, Bartosiewicz L, Gardeisen A, Kanstrup M, Maier U, Marinova E, Ninov L, Schäfer M, Stephan E (2013) Crop manuring and intensive land management by Europe's first farmers. Proc Nat Acad Sci USA 110:12589-12594. https://doi.org/10.1073/pnas.1305918110

Bonsall C (1981) The coastal factor in the Mesolithic settlement of Northwest England. In: Gramsch B (ed) Mesolithikum in Europa. Deutscher Verlag, Berlin, pp 451-472

Bonsall C, Boroneant A, Evatt A, Soficaru A, Nica C, Bartosiewicz L, Cook GT, Higham TFG, Pickard C (2016) The 'Clisurean' finds from Climente II cave, Iron Gates, Romania. Quat Int 423:303314. https://doi.org/10.1016/j.quaint.2015.12.017

Bonsall C, Cook G, Pickard C, McSweeney K, Bartosiewicz L (2009) Dietary trends at the Mesolithic-Neolithic transition in North-West Europe. In: Crombé P, Van Strydonck M, Sergant J, Boudin M, Bats $\mathrm{M}$ (eds) Chronology and evolution within the Mesolithic of NorthWest Europe. Cambridge Scholars Publishing, Newcastle-uponTyne, pp 517-539

Bonsall C, Cook G, Pickard C, McSweeney K, Sayle K, Bartosiewicz L, Radovanovic I, Higham T, Soficaru A, Boroneant A (2015) Food for thought: re-assessing Mesolithic diets in the Iron Gates. Radiocarbon 57:689-699. https://doi.org/10.2458/azu rc.57.18440

Bonsall C, Lennon R, McSweeney K, Stewart C, Harkness D, Boroneant V, Bartosiewicz L, Payton R, Chapman J (1997) Mesolithic and early Neolithic in the Iron Gates: a palaeodietary perspective. J Eur Archaeol 5:50-92. https://doi.org/10.1179/ 096576697800703575

Bownes J (2018) Reassessing the Scottish Mesolithic-Neolithic transition: questions of diet and chronology. Unpublished $\mathrm{PhD}$ thesis. University of Glasgow

Bownes JM, Ascough PL, Cook GT, Murray I, Bonsall C (2017) Using stable isotopes and a Bayesian mixing model (FRUITS) to investigate diet at the early Neolithic site of carding Mill Bay, Scotland. Radiocarbon 59:1275-1294. https://doi.org/10.1017/RDC.2017.39

Charlton S, Alexander M, Collins M, Milner N, Mellars P, O'Connell TC, Stevens R, Craig OE (2016) Finding Britain's last hunter-gatherers: a new biomolecular approach to 'unidentifiable' bone fragments utilising bone collagen. J Archaeol Sci 73:55-61. https://doi.org/ 10.1016/j.jas.2016.07.014

Chisholm BS, Nelson DE, Schwarcz HP (1982) Stable carbon ratios as a measure of marine versus terrestrial protein in ancient diets. Science 216:1131-1132. https://doi.org/10.1126/science.216.4550.1131

Churchill DM (1963) A report on the pollen analyses of the muds from the medulla tissues of two fossil human skeletons: Tilbury man and Thatcham man. Proceedings of the Prehistoric Society 29:427-428. : https://doi.org/10.1017/S0079497X00015474

Cleyet-Merle JJ (1990) La préhistoire de la pêche. Editions Errance, Paris

Cordain L, Brand Miller J, Eaton SB, Mann N, Holt SHA, Speth JD (2000) Plant-animal subsistence ratios and macronutrient energy estimations in worldwide hunter-gatherer diets. Am J Clin Nutr 71:682-692. https://doi.org/10.1093/ajcn/71.3.682
Crowley BE, Carter ML, Karpanty SM, Zihlman AL, Koch PL, Dominy $\mathrm{NJ}$ (2010) Stable carbon and nitrogen isotope enrichment in primate tissues. Oecologia 164:611-626. https://doi.org/10.1007/s00442010-1701-6

DeNiro MJ, (1985) Postmortem preservation and alteration of in-vivo bone collagen isotope ratios in relation to paleodietary reconstruction. Nature 317:806-809

DeNiro MJ, Epstein S (1978) Influence of diet on the distribution of carbon isotopes in animals. Geochim Cosmochim Acta 42:495-506

DeNiro MJ, Epstein S (1981) Influence of diet on the distribution of nitrogen isotopes in animals. Geochim Cosmochim Acta 45:341351. https://doi.org/10.1016/0016-7037(81)90244-1

Drucker D, Bocherens H (2004) Carbon and nitrogen stable isotopes as tracers of change in diet breadth during Middle and Upper Palaeolithic in Europe. Int J Osteoarchaeol 14:162-177. https:// doi.org/10.1002/oa.753

Drucker D, Bocherens H, Bridault A, Billiou D (2003) Carbon and nitrogen isotopic composition of red deer (Cervus elaphus) collagen as a tool for tracking palaeoenvironmental change during the late-glacial and Early Holocene in the northern Jura (France). Palaeogeogr Palaeoclimatol Palaeoecol 195:375-388. https://doi.org/10.1016/ S0031-0182(03)00366-3

Drucker DG, Naito YI, Jerardino A (2016) Aquatic resource exploitation by prehistoric humans. J Archaeol Sci Rep 6:621-622. https://doi. org/10.1016/j.jasrep.2015.12.021

Drucker DG, Valentin F, Thevenet C, Mordant D, Cottiaux R, Delsate D, van Neer W (2018) Aquatic resources in human diet in the late Mesolithic in northern France and Luxembourg: insights from carbon, nitrogen and sulphur isotope ratios. Archaeol Anthropol Sci 10: 351-368. https://doi.org/10.1007/s12520-016-0356-6

Eide M, Olsen A, Ninnemann US, Eldevik T (2017) A global estimate of the full oceanic ${ }^{13} \mathrm{C}$ Suess effect since the preindustrial. Glob Biogeochem Cycles 31:492-514. https://doi.org/10.1002/ 2016GB005472

Fernandes R, Grootes PM, Nadeau M-J, Nehlich O (2015) Quantitative diet reconstruction of a Neolithic population using a Bayesian mixing model (FRUITS): the case study of Ostorf (Germany). Am J Phys Anthropol 158:325-340. https://doi.org/10.1002/ajpa.22788

Fernandes R, Millard AR, Brabec M, Nadeau M-J, Grootes P (2014) Food reconstruction using isotopic transferred signals (FRUITS): a Bayesian model for diet reconstruction. PLoS One 9(2):e87436. https://doi.org/10.1371/journal.pone.0087436

Finlay JC, Kendall C (2007) Stable isotope tracing of temporal and spatial variability in organic matter sources to freshwater ecosystems. In: Michener R, Lajtha K (eds) Stable isotopes in ecology and environmental science. Blackwell, Malden/Oxford/Victoria, pp 283-333

Fischer A, Heinemeier J, Richards M, Heinemeier J, Sveinbjörnsdóttir ÁE, Bennike P (2007) Coast-inland mobility and diet in the Danish Mesolithic and Neolithic: evidence from stable isotope values of humans and dogs. J Archaeol Sci 34(12):2125-2150

Fuller BT, Fuller JL, Harris DA, Hedges REM (2006) Detection of breastfeeding and weaning in modern human infants with carbon and nitrogen stable isotope ratios. Am J Phys Anthropol 129:279293. https://doi.org/10.1002/ajpa.20249

Germain LR, McCarthy MD, Koch PL, Harvey JT (2012) Stable carbon and nitrogen isotopes in multiple tissues of wild and captive harbor seals (Phoca vitulina) off the California coast. Mar Mammal Sci 28: 542-560. https://doi.org/10.1111/j.1748-7692.2011.00516.x

Gillespie R, Gowlett JAJ, Hall ET, Hedges REM, Perry C (1985) Radiocarbon dates from the oxford ams system: archaeometry datelist 2. Archaeometry 27(2):237-246

Gowlett JAJ, Hall ET, Hedges REM, Perry C (1986) Radiocarbon dates from the Oxford AMS system: Archaeometry datelist 2. Archaeometry 27:237-246. https://doi.org/10.1111/j.1475-4754. 1985.tb00367.x 
Guiry E (2019) Complexities of stable carbon and nitrogen isotope biogeochemistry in ancient freshwater ecosystems: implications for the study of past subsistence and environmental change. Front Ecol Evol 7. https://doi.org/10.3389/fevo.2019.00313

Hedges REM, Housley RA, Law IA, Perry C, Gowlett JAJ (1987) Radiocarbon dates from the Oxford AMS system: Archaeometry datelist 6. Archaeometry 29:289-306. https://doi.org/10.1111/j. 1475-4754.1987.tb00421.x

Hedges REM, Pettitt PB, Bronk Ramsey C, van Klinken GJ (1996) Radiocarbon dates from the Oxford AMS system: Archaeometry datelist 22. Archaeometry 38:391-415. https://doi.org/10.1111/j. 1475-4754.1996.tb00785.x

Hedges REM, Clement JG, Thomas CDL, O'Connell TC (2007) Collagen turnover in the adult femoral mid-shaft: modeled from anthropogenic radiocarbon tracer measurements. Am J Phys Anthropol 133:808-816

Hedges REM, Housley RA, Bronk Ramsey C, Van klinken GJ (1994) Radiocarbon dates from the oxford ams system: archaeometry datelist 18 . Archaeometry 36(2):337-374

Hill TCB, Woodland WA, Spencer CD, Marriott SB, Case DJ, Catt JA (2008) Devensian Late-glacial environmental change in the Gordano Valley, North Somerset, England: a rare archive for Southwest Britain. J Limnol 40:431-444

Hobson KA, Schell DM, Renouf D, Noseworthy E (1996) Stable carbon and nitrogen isotopic fractionation between diet and tissues of captive seals: implications for dietary reconstructions involving marine mammals. Can J Fish Aquat Sci 53:528-533. https://doi.org/10. 1139/cjfas-53-3-528

Ince J (1996) Late-glacial and Early Holocene vegetation of Snowdonia. New Phytologist 132:343-353 https://www.jstor.org/stable/ 2558457. Accessed 23 May 2019

Jacobi RM, Higham TFG (2009) The early Lateglacial re-colonization of Britain: new radiocarbon evidence from Gough's cave, Southwest England. Quat Sci Rev 28:1895-1913. https://doi.org/10.1016/j. quascirev.2009.03.006

Katzenberg MA (2000) Stable isotope analysis: a tool for studying past diet, demography, and life history. In: Katzenberg MA, Saunders SR (eds) Biological anthropology of the human skeleton. Wiley-Liss, Hoboken, pp 413-442

Keeling CD (1979) The suess effect: 13Carbon-14Carbon interrelations. Environ Int 2(4-6):229-300

Logan JM, Jardine TD, Miller TJ, Bunn SE, Cunjak RA, Lutcavage ME (2008) Lipid corrections in carbon and nitrogen stable isotope analyses: comparison of chemical extraction and modelling methods. J Anim Ecol 77:838-846. https://doi.org/10.1111/j.1365-2656.2008. 01394.x

Long SP (1983) $\mathrm{C}_{4}$ photosynthesis at low temperatures. Plant Cell Environ 6:345-363. https://doi.org/10.1111/1365-3040. ep11612141

Maberly SC, Raven JA, Johnston AM (1992) Discrimination between ${ }^{12} \mathrm{C}$ and ${ }^{13} \mathrm{C}$ by marine plants. Oecologia $91: 481-492$. https://doi. org/10.1007/BF00650320

Meadows J, Lozovskaya O, Bondetti M, Drucker DG, Moisey V (2019) Human palaeodiet at Zamostje 2, Central Russia: results of radiocarbon and stable isotope analyses. Quat Int 541:89-103. https://doi. org/10.1016/j.quaint.2019.07.017

Meiklejohn C, Chamberlain TA, Schulting RJ (2011) Radiocarbon dating of Mesolithic human remains in Great Britain. Mesolithic Miscellany 21:20-58

Milner N, Craig O (2012) Isotope analyses. In: Saville A, Hardy K, Miket RF, Ballin TB (eds) An Corran, Staffin, Skye: a rockshelter with Mesolithic and later occupation. Council for British Archaeology, York, pp 77-79. https://doi.org/10.5284/1017938

Montgomery J, Beaumont J, Jay M, Keefe K, Gledhill AR, Cook GT, Dockrill SJ, Melton ND (2013) Strategic and sporadic marine consumption at the onset of the Neolithic: increasing temporal resolution in the isotope evidence. Antiquity 87:1060-1072. https://doi.org/10.1017/S0003598X00049863

Nadelhoffer KJ, Fry B (1994) Nitrogen isotope studies in forest ecosystems. In: Lajtha K, Michener R (eds) Stable isotopes in ecology. Blackwell, Oxford, pp 22-44

Nehlich O, Borić D, Stefanović S, Richards MP (2010) Sulphur isotope evidence for freshwater fish consumption: a case study from the Danube gorges, SE Europe. J Archaeol Sci 37:1131-1139. https:// doi.org/10.1016/j.jas.2009.12.013

Newsome SD, Clementz MT, Koch PL (2010) Using stable isotope biogeochemistry to study marine mammal ecology. Mar Mammal Sci 26:509-572. https://doi.org/10.1111/j.1748-7692.2009.00354.x

Nichol RK, Wild CJ (1984) "Numbers of individuals" in faunal analysis: the decay of fish bone in archaeological sites. J Archaeol Sci 11:3551. https://doi.org/10.1016/0305-4403(84)90040-2

O'Connell TC, Kneale CJ, Tasevska N, Kuhnle GGC (2012) The dietbody offset in human nitrogen isotopic values: a controlled dietary study. Am J Phys Anthropol 49:426-434. https://doi.org/10.1002/ ajpa. 22140

Parnell AC, Inger R, Bearhop S, Jackson AL (2014) Source partitioning using stable isotopes. PLoS One 5(3):e9672. https://doi.org/10. 1371/journal.pone.0009672

Phillips DL, Newsome SD, Gregg JW (2005) Combining sources in stable isotope mixing models. Oecologia 144:520-527. https://doi. org/10.1007/s00442-004-1816-8

Phillips DL, Koch PL (2002) Incorporating concentration dependence in stable isotope mixing models. Oecologia 130:114-125. https://doi. org/10.1007/s004420100786

Pickard C, Bonsall C (2004) Deep sea fishing in the European Mesolithic: fact or fantasy? Eur J Archaeol 7:273-290. https://doi.org/10.1177/ $1461957104056504 \mathrm{P}$

Pinnegar JK, Polunin NVC (1999) Differential fractionation of $\delta^{13} \mathrm{C}$ and $\delta^{15} \mathrm{~N}$ among fish tissues: implications for the study of trophic interactions. Funct Ecol 13:225-231. https://doi.org/10.1046/j.13652435.1999.00301.x

Post DM, Layman CA, Arrington DA, Takimoto G, Quattrochi J, Montaña CG (2007) Getting to the fat of the matter: models, methods and assumptions for dealing with lipids in stable isotope analyses. Oecologia 152:179-189. https://doi.org/10.1007/s00442006-0630-x

Preston PR, Kador T (2018) Approaches to interpreting Mesolithic mobility and settlement in Britain and Ireland. J World Prehist 31:321345. https://doi.org/10.1007/s10963-018-9118-y

Ricca MA, Miles AK, Anthony RG, Deng X, Hung SSO (2007) Effect of lipid extraction on analyses of stable carbon and stable nitrogen isotopes in coastal organisms of the Aleutian archipelago. Can J Zool 85:40-48. https://doi.org/10.1139/Z06-187

Richards MP, Hedges REM, Jacobi R, Current A, Stringer C (2000) Gough's Cave and Sun Hole Cave human stable isotope values indicate a high protein diet in the British Upper Palaeolithic. J Archaeol Sci 27:1-3. https://doi.org/10.1006/jasc.1999.0520

Richards MP, Jacobi R, Cook J, Pettitt PB, Stringer CB (2005) Isotope evidence for the intensive use of marine foods in the Late Upper Palaeolithic. J Hum Evol 49:390-394. https://doi.org/10.1016/j. jhevol.2005.05.002

Richards MP, Karavanić I, Pettitt P, Miracle P (2015) Isotope and faunal evidence for high levels of freshwater fish consumption by late glacial humans at the Late Upper Palaeolithic site of Šandalja, II, Istria, Croatia. J Archaeol Sci 61:204-212. https://doi.org/10.1016/j. jas.2015.06.008

Richards MP, Mellars P (1998) Stable isotopes and the seasonality of the Oronsay middens. Antiquity 72:178-184. https://doi.org/10.1017/ S0003598X00086373

Robson HK, Andersen SH, Clarke L, Craig E, Gron KJ, Jones AKG, Milner N, Price TD, Ritchie K, Mirosława Z-K, Heron C (2016) Carbon and nitrogen stable isotope values in freshwater, brackish 
and marine fish bone collagen from Mesolithic and Neolithic sites in central and northern Europe. Environ Archaeol 21:105-118. https:// doi.org/10.1179/1749631415Y.0000000014

Schoeninger MJ, DeNiro MJ (1984) Nitrogen and carbon isotopic composition of bone collagen from marine and terrestrial animals. Geochim Cosmochim Acta 48:625-639. https://doi.org/10.1016/ 0016-7037(84)90091-7

Schoeninger MJ (2010) Diet reconstruction and ecology using stable isotope ratios. In: Larsen CS (ed) A companion to biological anthropology. Wiley-Blackwell, Chichester, pp 445-464

Schulting RJ, Fibiger L, Macphail RI, McLaughlin R, Murray E, Price CR, Walker EA (2013) Mesolithic and Neolithic human remains from Foxhole Cave, Gower, South Wales. Antiqu J 93:1-23. https://doi.org/10.1017/S000358151300019X

Schulting R, Richards MP (2002a) Finding the coastal Mesolithic in Southwest Britain: AMS dates and stable isotope results on human remains from Caldey Island, South Wales. Antiquity 76:1011-1025. https://doi.org/10.1017/S0003598X00091821

Schulting R, Richards MP (2002b) The wet, the wild and the domesticated: the Mesolithic-Neolithic transition on the west coast of Scotland. Antiquity 5:147-189. https://doi.org/10.1177/ 14619571020050020201

Schulting R, Richards MP (2009) Dogs, divers, deer and diet. Stable isotope results from Star Carr and a response to Dark. J Archaeol Sci 36:498-503. https://doi.org/10.1016/j.jas.2008.09.035

Selva N, Hobson K, Cortés-Avizanda A, Zalewski A, Donázar JA (2012) Mast pulses shape trophic interactions between fluctuating rodent populations in a primeval forest. PLoS One 7(12):e51267. https:// doi.org/10.1371/journal.pone.0051267

Shennan I, Bradley S, Milne G, Brooks A, Bassett S, Hamilton S (2006) Relative sea-level changes, glacial isostatic modelling and ice-sheet reconstructions from the British Isles since the Last Glacial Maximum. J Quat Sci 21:585-599. https://doi.org/10.1002/jqs.1049

Sotiropoulos MA, Tonn WM, Wassenaar LI (2004) Effects of lipid extraction on stable carbon and nitrogen isotope analyses of fish tissues: potential consequences for food web studies. Ecol Freshw Fish 13:155-160. https://doi.org/10.1111/j.1600-0633.2004.00056.x

Stevens R, Hedges REM (2004) Carbon and nitrogen stable isotope analysis of northwest European horse bone and tooth collagen, 40,000 BP-present: palaeoclimatic interpretations. Quat Sci Rev 23:977991. https://doi.org/10.1016/j.quascirev.2003.06.024

Stevens RE, Jacobi R, Higham TFG (2010) Reassessing the diet of Upper Palaeolithic humans from Gough's Cave and Sun Hole, Cheddar
Gorge, Somerset, UK. J Archaeol Sci 37:52-61. https://doi.org/10. 1016/j.jas.2009.08.019

Stevens RE, Jacobi R, Street M, Germonpré M, Conard NJ, Münzel SC, Hedges REM (2008) Nitrogen isotope analyses of reindeer (Rangifer tarandus), 45,000 BP to 9,000 BP: palaeoenvironmental reconstructions. Palaeogeogr Palaeoclimatol Palaeoecol 262:32-45. https://doi.org/10.1016/j.palaeo.2008.01.019

Thode HD (1991) Sulphur isotopes in nature and the environment: an overview. In: Krouse HR, Grinenko VA (eds) Stable isotopes in the assessment of natural and anthropogenic sulphur in the environment, vol 43. Scientific Committee on Problems of the Environment, pp 1-26

Thornton SF, McManus J (1994) Application of organic carbon and nitrogen stable isotope and $\mathrm{C} / \mathrm{N}$ ratios as source indicators of organic matter provenance in estuarine systems: evidence from the Tay Estuary, Scotland. Estuar Coast Shelf Sci 38:219-233. https://doi. org/10.1006/ecss.1994.1015

Tykot RH (2004) Stable isotopes and diet: you are what you eat. In: Martini M, Milazzo M, Piacentini M (eds) Physics methods in archaeometry, Proceedings of the International School of Physics "Enrico Fermi" course CLIV. IOS Press, Amsterdam, pp 433-444

USDA Nutrient Database. https://ndb.nal.usda.gov/. Accessed 12 Feb 2019

van der Merwe NJ, Medina E (1991) The canopy effect, carbon isotope ratios and foodwebs in Amazonia. J Archaeol Sci 18:249-259. https://doi.org/10.1016/0305-4403(91)90064-V

Vogel JC, Van Der Merwe NJ (1977) Isotopic evidence for early maize cultivation in New York State. Am Antiq 42:238-242 https://www. jstor.org/stable/278984

Waters-Rist AL, Katzenberg MA (2010) The effect of growth on stable nitrogen isotope ratios in subadult bone collagen. Int $\mathrm{J}$ Osteoarchaeol 20:172-191. https://doi.org/10.1002/oa.1017

Watkins BE, Witham JH, Ullrey DE, Watkins DJ, Jones JM (1991) Body composition and condition evaluation of white-tailed deer fawns. J Wildl Manag 55:39-51 https://www.jstor.org/stable/3809239

von Wirén N, Gazzarrini S, Frommer WB (1997) Regulation of mineral nitrogen uptake in plants. Plant Soil 196:191-199. https://doi.org/ 10.1023/A: 1004241722172

Publisher's note Springer Nature remains neutral with regard to jurisdictional claims in published maps and institutional affiliations. 